\title{
Semiclassical response of disordered conductors: Extrinsic carrier velocity and spin and field-corrected collision integral
}

\author{
Rhonald Burgos Atencia $\odot,{ }^{1}$ Qian Niu, ${ }^{2,3}$ and Dimitrie Culcer, ${ }^{4} *$ \\ ${ }^{1}$ Facultad de Ingenierías, Departamento de Ciencias Básicas, Universidad del Sinú, Carrera 1w No. 38-153, 4536534, Montería, \\ Córdoba 230002, Colombia \\ ${ }^{2}$ International Center for Quantum Design of Functional Materials, Hefei National Laboratory for Physical Sciences at the Microscale, \\ University of Science and Technology of China, Hefei, Anhui 230026, China \\ ${ }^{3}$ School of Physics, University of Science and Technology of China, Hefei, Anhui 230026, China \\ ${ }^{4}$ School of Physics, The University of New South Wales, Sydney, New South Wales 2052, Australia
}

(Received 30 September 2021; accepted 24 November 2021; published 3 January 2022)

\begin{abstract}
The semiclassical equations of motion are widely used to describe carrier transport in conducting materials. Nevertheless, the substantial challenge of incorporating disorder systematically into the semiclassical model persists, leading to quantitative inaccuracies and occasionally erroneous predictions for the expectation values of physical observables. To address this issue, in this paper we provide a general prescription for reformulating the semiclassical equations of motion for carriers in disordered conductors by taking the quantum mechanical density matrix as the starting point. We focus on the case when only external electric fields are present, without magnetic fields, and the disorder potential is spin independent. The density matrix approach allows averaging over impurity configurations, and the trace of the velocity operator with the disorder-averaged density matrix can be reinterpreted as the semiclassical velocity weighted by the Boltzmann distribution function. Through this rationale the well-known intrinsic group and anomalous velocities are trivially recovered, while we demonstrate the existence of an extrinsic velocity, namely, a disorder correction to the semiclassical velocity of Bloch electrons, mediated by the interband matrix elements of the Berry connection. A similar correction is present in the nonequilibrium expectation value of the spin operator, contributing to spin-orbit torques. To obtain agreement with diagrammatic approaches, the scattering term in the Boltzmann equation must be corrected to first order in the applied electric field, and the Boltzmann equation itself must be solved up to subleading order in the disorder potential. Our prescription ensures that all vertex corrections present in diagrammatic treatments are taken into account, and to illustrate this, we discuss model cases in topological insulators, including the anomalous Hall effect as well as spin-orbit torques.
\end{abstract}

DOI: 10.1103/PhysRevResearch.4.013001

\section{INTRODUCTION}

Carrier transport in extended conductors is an inherently semiclassical phenomenon, requiring an effective singleparticle description as well as averaging over real space and momentum space degrees of freedom. The semiclassical model [1], which takes these ingredients as natural building blocks, has been a staple of transport theory for the best part of a century [2]. In recent years, aside from its well-established application to the anomalous Hall effect and dynamics in magnetic systems [3-7], it has frequently been used to describe transport in electric and magnetic fields in systems with nontrivial topological textures [8-27], including nonlinear electromagnetic responses [28-33], and has recently

\footnotetext{
*d.culcer@unsw.edu.au

Published by the American Physical Society under the terms of the Creative Commons Attribution 4.0 International license. Further distribution of this work must maintain attribution to the author(s) and the published article's title, journal citation, and DOI.
}

found substantial applications in computational approaches to nonequilibrium physics [34-48]. In addition to its profound physical insight, broad applicability, and relative simplicity, the semiclassical method naturally accounts for topological effects and enables a clear identification of Fermi surface and Fermi sea contributions to transport [49-52].

The central idea of the semiclassical model is the separation between the dynamics of individual carriers and the carrier distribution. Carrier dynamics between collisions are described by the semiclassical equations of motion, which do not incorporate disorder, while collisions are taken into account through the Boltzmann equation and affect solely the distribution function, inducing changes in the occupation of quantum states [2]. The semiclassical velocity, originally assumed to be simply the band group velocity, is now known to incorporate a transversal anomalous component linear in the driving electric field and proportional to the Berry curvature $\boldsymbol{\Omega}_{m}$ of a given band $m[1,53]$. Although written in terms of the curvature for a single band, this anomalous velocity includes interband coherence effects and is associated with band mixing by an electric field [50]. The anomalous velocity lies at the heart of the quantum Hall effect and of the intrinsic contribu- 
tion to the anomalous Hall effect, together with its quantized counterpart. In recent years, however, it has been realized that disorder itself leads to band-mixing effects which are not captured by a naive solution to the Boltzmann equation and are challenging to include in the conventional wave-packet description. This is primarily because the standard treatment of disorder involves averaging over configurations, and this cannot be done at the level of the wave function. It is well established that a naive application of the semiclassical model to the anomalous and spin-Hall effects in disordered systems makes inaccurate predictions [54-60] and that the simple relaxation time approximation applied solely to the Boltzmann equation does not capture the full underlying physics of coherent scattering off the random disorder potential. Indeed, the role of disorder in the anomalous Hall effect [61-65] and its relationship to semiclassical dynamics remains an intensely researched topic [35,39,66-73].

Nevertheless, since transport is fundamentally semiclassical, all transport-related quantities must be expressible in semiclassical terms. The assumptions behind wave-packet dynamics and diagrammatic approaches are the same: External fields are treated classically and are assumed to be slowly varying in space, a separation is made between scattering processes and the dynamics between scattering events, and the calculation is performed in the regime $\epsilon_{F} \tau / \hbar \gg 1$, where $\epsilon_{F}$ is the Fermi energy and $\tau$ is the momentum relaxation time. Recent work has investigated strategies for incorporating the findings of diagrammatic linear response theory into semiclassical dynamics. Sinitsyn and co-workers introduced a spin-dependent coordinate shift into the position operator and identified a side-jump velocity [74-76]. These quantities, however, are difficult to work with, since a spin-dependent position operator introduces complications in keeping track of relevant contributions, as well as in the velocity operator. $\mathrm{Xiao}, \mathrm{Niu}$, and co-workers showed that agreement is obtained with diagrammatic approaches if all semiclassical quantities are dressed by disorder, including a disorder-dressed Berry curvature $[77,78]$. However, the wave-packet approach to semiclassical transport is well defined only for the disorderfree case and is challenging to extend to disordered systems since averaging over disorder configurations cannot be done for wave packets.

In light of the above, in this paper we formulate the semiclassical equations of motion for Bloch electrons so as to include disorder, starting from the quantum mechanical density matrix [50], in the process making a connection to Green's functions approaches. A strong motivation for our work is the recent surge in applications of the semiclassical model in computational studies of transport in topological materials [34-48]. Our primary aim is to provide a straightforward method to incorporate disorder into such computational strategies once a model of disorder is chosen, together with a set of self-contained equations serving this purpose. The use of the density matrix to treat transport phenomena in the presence of disorder has been well established since the seminal paper by Luttinger [62]. Nevertheless, the nonequilibrium density matrix in the presence of disorder is a formidable quantity, and a straightforward application of Luttinger's expressions to realistic systems for explicit computations is not possible. The main complexity in the methodology of Ref. [62] is the attempt to isolate simultaneously the part of the density matrix diagonal in both the wave vector and the band index. On the other hand, taking the density matrix as the starting point [79] allows one to average over disorder configurations, something that cannot be done using a wave function. In this paper, in contrast to Luttinger [62], we introduce the notion of a disorder average from the outset and immediately decompose the reduced density matrix into a disorder-averaged part and a fluctuating part. The disorder average restores translational periodicity, and as a consequence the disorderaveraged density matrix in a noninteracting system is diagonal in wave vector in the crystal momentum representation. In this way, the process of isolating the band-diagonal and wavevector-diagonal part of the density matrix becomes far more transparent and tractable. By considering band-diagonal and band-off-diagonal projections of the disorder-averaged density matrix we account for the role of spin-charge coupling in the dynamical system [80]. We demonstrate that disorder affects not only the state occupation but also the semiclassical equations of motion and that it generates a correction to the velocity that accounts for band mixing mediated by the Berry connection and disorder. This approach enables one to distinguish disorder effects on the distribution function from disorder effects on carrier dynamics; yet it entails a change in one's point of view so as to regard the semiclassical equations as describing carrier propagation averaged over many disorder scattering events. The carrier undergoes transitions between bands as it scatters, and its trajectory can be determined by averaging over impurity configurations. Whereas the equation of motion for the wave vector $\boldsymbol{k}$ follows trivially from the time derivative of the momentum operator, our central result is the revised semiclassical equation of motion for the position of a carrier in band $m$, with dispersion $\epsilon_{m}$, propagating under the action of an electric field $\boldsymbol{E}$ in the presence of disorder

$$
\dot{\boldsymbol{r}}_{m}=\frac{1}{\hbar} \frac{\partial \epsilon_{m}}{\partial \boldsymbol{k}}+\frac{\boldsymbol{e}}{\hbar} \boldsymbol{E} \times \boldsymbol{\Omega}_{m}+\boldsymbol{\beta}_{\boldsymbol{k}}^{m} .
$$

We identify a new contribution to the velocity, which we term the extrinsic velocity $\boldsymbol{\beta}_{\boldsymbol{k}}^{m}$, defined as

$$
\boldsymbol{\beta}_{\boldsymbol{k}}^{m}=\frac{1}{2 \pi \hbar} \int_{-\infty}^{\infty} d \epsilon\left\langle\left[U, G_{0}^{A}(\epsilon)\left[U, \mathcal{R}^{\prime}\right] G_{0}^{R}(\epsilon)\right]\right\rangle_{\boldsymbol{k}}^{m m}
$$

where $\mathcal{R}^{\prime}$ represents the interband Berry connection with matrix elements $\mathcal{R}_{\boldsymbol{k}}^{\prime m n}=i\left\langle u_{\boldsymbol{k}}^{m} \mid \nabla_{\boldsymbol{k}} u_{\boldsymbol{k}}^{n}\right\rangle_{m \neq n}$ and $\left|u_{\boldsymbol{k}}^{m}\right\rangle$ is the lattice-periodic part of the Bloch wave function. The extrinsic velocity $\boldsymbol{\beta}_{\boldsymbol{k}}^{m}$ is proportional to the disorder strength, which is typically quantified by the impurity density $n_{i}$, scattering potential strength $u_{0}^{2}$, or alternatively $1 / \tau$, where $\tau$ is the characteristic scattering time. Note that this correction to the velocity is independent of the applied electric field. In the semiclassical regime, in which $\epsilon_{F} \tau / \hbar \gg 1$, where $\epsilon_{F}$ is the Fermi energy, this quantity is of first order in the small parameter $\hbar /\left(\epsilon_{F} \tau\right)$. Nevertheless, to obtain its contribution to the current, it is necessary to multiply it by the large change in the Boltzmann distribution $\propto \tau$, such that the final result appears to be formally independent of disorder, of order $\left[\hbar /\left(\epsilon_{F} \tau\right)\right]^{0}$. The expression for $\boldsymbol{\beta}_{\boldsymbol{k}}^{m}$ is analogous to the customary scattering term in the Born approximation, except the distribution function is replaced by the band-off-diagonal elements of the Berry connection. Since only the band-off-diagonal elements 
of the Berry connection appear, $\boldsymbol{\beta}_{k}^{m}$ is by construction gauge covariant. In general, $\boldsymbol{\beta}_{k}^{m}$, being independent of applied fields, can be thought of as a disorder-dependent correction to the semiclassical band velocity, or a random interband walk on the Fermi surface. We find that $\boldsymbol{\beta}_{k}^{m}$ is nonzero in systems in which time-reversal symmetry is broken by, e.g., a magnetization. Whereas $\boldsymbol{\beta}_{k}^{m}$ is similar to the side jump as defined in Ref. [81], unlike Ref. [81], the present formalism does not employ coordinate shifts, so that the formal and physical position operators coincide. Furthermore, unlike Ref. [77], the Berry curvature is the same as in the clean system, rather than being dressed by disorder. Importantly, we show that the scattering term in the Boltzmann equation, needed to determine the effective distribution function, acquires a correction to first order in the electric field, which is equivalent to a gradient expansion in the electrostatic potential. In addition, the Boltzmann equation needs to be solved up to the subleading order in the impurity strength, in order to incorporate processes customarily termed skew scattering and side jump $[63,64,82]$. The method we present here also enables us to calculate spin densities using the semiclassical model and obtain accurate results for spin-orbit torques. In order to accomplish this the bare spin expectation value needs to be supplemented with an electric-field contribution, and we find an analogous quantity to $\boldsymbol{\beta}_{k}^{m}$ in the spin expectation value.

More generally, we present a prescription for mapping steady-state expectation values onto the semiclassical model by expressing traces purely in terms of the band-diagonal elements of the density matrix. Since in linear response theory all expectation values are traced back to the equilibrium density matrix, which is band diagonal, they can all be recast in terms of semiclassical quantities. The band-diagonal elements of the density matrix represent the Boltzmann distribution, which can be evaluated from the much simpler Boltzmann equation. In fact, we will argue briefly in the latter part of this paper that linear response theories can be thought of as a family tree with its roots in the quantum Liouville equation: The Kubo approach is the integral formulation, and the quantum kinetic, or quantum Boltzmann, approach is the integrodifferential formulation, while the semiclassical model is an offshoot of the latter, which arises as a result of an additional separation between the carrier dynamics and distribution. Sharing a common origin, these methods yield equivalent results, and, in particular, vertex corrections present in diagrammatic approaches have straightforward equivalents in the semiclassical language. The blueprint presented in this paper can be used in the future to incorporate electron-electron interactions into computational approaches in a mean-field picture.

This paper builds on earlier work on the density matrix theory of transport $[50,83]$, which introduced the notion of the impurity average and the description of electric and magnetic fields within the density matrix framework. These concepts are reviewed in part in Sec. III. The present paper, which employs a similar methodology in order to construct a disorder-averaged semiclassical model of transport, can be regarded as the third paper in this sequence.

The outline of this paper is as follows. In Sec. II we introduce the Hamiltonian and the model of disorder. In Sec. III we review linear response theory based on the density matrix and introduce the electric-field correction to the collision term. The main equations in this section are the following: Eq. (34), which provides a general relation to calculate the complete subleading distribution function, namely, a distribution function due to skew scattering plus an anomalous distribution function due to an electric-field correction of the collision integral, and Eq. (36) along with Eq. (38), which are explicit expressions to solve the abovementioned subleading function. Next, in Sec. IV we outline the general methodology for deriving the semiclassical equations of motion from the quantum kinetic equation and discuss also disorder effects on spin expectation values. The main equations in this section are Eqs. (47) and (61), which are explicit expressions to compute the new extrinsic velocity and the new extrinsic spin expectation value. In Sec. V we analyze the relation among different linear response methodologies commonly used to calculate transport coefficients. In Sec. VI we discuss at length two model examples, the anomalous Hall effect and spin-orbit torques in magnetic topological insulators. We end with a summary and conclusions.

\section{MODEL HAMILTONIAN}

We consider a Hamiltonian of the form

$$
H=H_{0}+V(\boldsymbol{r})+U(\boldsymbol{r}),
$$

where $H_{0}$ is the low-energy effective band Hamiltonian, in principle assumed to include the Zeeman interaction with an external magnetic field, $V(\boldsymbol{r})$ is the electrostatic potential, and $U=U(\boldsymbol{r})$ represents the disorder scattering potential. We emphasize that the Hamiltonian is always Hermitian. NonHermitian systems were considered in Ref. [84].

We work in the crystal momentum representation $|m, \boldsymbol{k}\rangle=$ $e^{i k \cdot r}\left|u_{k}^{m}\right\rangle$. The matrix elements of a scalar disorder potential $U(\boldsymbol{r})$ are given by the equation

$$
U_{\boldsymbol{k} \boldsymbol{k}^{\prime}}^{m m^{\prime}}=\left\langle u_{\boldsymbol{k}}^{m} \mid u_{\boldsymbol{k}^{\prime}}^{m^{\prime}}\right\rangle \mathcal{U}_{\boldsymbol{q}},
$$

where we have defined the Fourier transform of the spatial function in $d$ dimensions

$$
\mathcal{U}_{\boldsymbol{q}}=\int d^{d} \boldsymbol{r} U(\boldsymbol{r}) e^{-i \boldsymbol{q} \cdot \boldsymbol{r}},
$$

with $\boldsymbol{q}=\boldsymbol{k}-\boldsymbol{k}^{\prime}$. The impurity average is defined by

$$
\left\langle U_{\boldsymbol{k} \boldsymbol{k}^{\prime}}^{m m^{\prime}} U_{\boldsymbol{k}^{\prime} \boldsymbol{k}}^{n^{\prime} n}\right\rangle=\left\langle u_{\boldsymbol{k}}^{m} \mid u_{\boldsymbol{k}^{\prime}}^{m^{\prime}}\right\rangle\left\langle u_{\boldsymbol{k}^{\prime}}^{n^{\prime}} \mid u_{\boldsymbol{k}}^{n}\right\rangle\left\langle\mathcal{U}_{\boldsymbol{q}} \mathcal{U}_{-\boldsymbol{q}}\right\rangle,
$$

with

$$
\left\langle\mathcal{U}_{q} \mathcal{U}_{-q}\right\rangle=\iint d^{d} \boldsymbol{r} d^{d} \boldsymbol{r}^{\prime}\left\langle U(\boldsymbol{r}) U\left(\boldsymbol{r}^{\prime}\right)\right\rangle e^{-i \boldsymbol{q} \cdot\left(\boldsymbol{r}-\boldsymbol{r}^{\prime}\right)},
$$

where $\langle\cdots\rangle$ refers to an average over impurity configurations. For concreteness we will use a model of disorder whose spatial correlation function is defined as

$$
\begin{gathered}
\langle U(\boldsymbol{r})\rangle=0, \\
\left\langle U(\boldsymbol{r}) U\left(\boldsymbol{r}^{\prime}\right)\right\rangle=u_{0}^{2} \delta\left(\boldsymbol{r}-\boldsymbol{r}^{\prime}\right) .
\end{gathered}
$$

Then, it follows that

$$
\left\langle U_{\boldsymbol{k} \boldsymbol{k}^{\prime}}^{m m^{\prime}} U_{\boldsymbol{k}^{\prime} \boldsymbol{k}}^{n^{\prime} n}\right\rangle=u_{0}^{2}\left\langle u_{\boldsymbol{k}}^{m} \mid u_{\boldsymbol{k}^{\prime}}^{m^{\prime}}\right\rangle\left\langle u_{\boldsymbol{k}^{\prime}}^{n^{\prime}} \mid u_{\boldsymbol{k}}^{n}\right\rangle,
$$

where $u_{0}^{2}$ is a parameter that takes into account the strength of the disorder potential. One can also resort to a model that 
makes explicit reference to the impurity density, as described in Ref. [50].

\section{QUANTUM KINETIC EQUATION}

In this section we give a brief presentation of the quantum kinetic equation in somewhat different language from that used in Ref. [50]. We note that similar density-matrix-based approaches have been used recently to describe carrier dynamics in the semiclassical regime [51,52]. The starting point is the quantum Liouville equation for the single-particle density operator $\rho$, namely,

$$
\frac{\partial \rho}{\partial t}+\frac{i}{\hbar}[H, \rho]=0 .
$$

For the sake of convenience, we introduce at this stage the free retarded Green's function

$$
G_{0}^{R}(t)=-i \theta(t) e^{-i t H_{0} / \hbar} .
$$

In the frequency domain,

$$
G_{0}^{R}(\epsilon)=-\frac{i}{\hbar} \int_{0}^{\infty} d t e^{-i H_{0} t / \hbar} e^{i \epsilon t / \hbar} e^{-\eta t},
$$

where we introduced the factor $e^{-\eta t}$ to ensure convergence. The advanced Green's function follows by Hermitian conjugation.

\section{A. Kinetic equation in equilibrium}

For the sake of simplicity, let us for now ignore the effect of the driving electric field in the kinetic equation. We decompose the density matrix as $\rho=\langle\rho\rangle+g_{0}$, where $\langle\rho\rangle$ is averaged over disorder configurations, while $g_{0}$ is the fluctuating part. Applying this decomposition to the quantum Liouville equation [50], we obtain for the disorder-averaged part in equilibrium

$$
\frac{\partial\langle\rho\rangle}{\partial t}+\frac{i}{\hbar}\left[H_{0},\langle\rho\rangle\right]+\frac{i}{\hbar}\left\langle\left[U, g_{0}\right]\right\rangle=0,
$$

while for the fluctuating part $g_{0}$ we find

$$
\frac{\partial g_{0}}{\partial t}+\frac{i}{\hbar}\left[H_{0}, g_{0}\right]+\frac{i}{\hbar}\left[U, g_{0}\right]-\frac{i}{\hbar}\left\langle\left[U, g_{0}\right]\right\rangle=-\frac{i}{\hbar}[U,\langle\rho\rangle] .
$$

In order to solve the kinetic equation for $\langle\rho\rangle$, we first solve Eq. (15) for $g_{0}$, and then we use it in Eq. (14). In the first Born approximation [50] we neglect the last two terms on the left-hand side of Eq. (15). We are left with

$$
\frac{\partial g_{0}}{\partial t}+\frac{i}{\hbar}\left[H_{0}, g_{0}\right]=-\frac{i}{\hbar}[U,\langle\rho\rangle] .
$$

Solving for the fluctuating part $g_{0}$,

$$
g_{0}=-\frac{i}{\hbar} \int_{0}^{\infty} d t^{\prime}\left[e^{-i H_{0} t^{\prime} / \hbar} U e^{i H_{0} t^{\prime} / \hbar},\langle\rho(t)\rangle\right] .
$$

In terms of Green's functions, $g_{0}$ can be expressed as

$$
g_{0}=\frac{1}{2 \pi i} \int_{0}^{\infty} d \epsilon\left[G_{0}^{R}(\epsilon) U G_{0}^{A}(\epsilon),\langle\rho(t)\rangle\right] .
$$

This solution is substituted into Eq. (14). We arrive at the equation

$$
\frac{\partial\langle\rho\rangle}{\partial t}+\frac{i}{\hbar}\left[H_{0},\langle\rho\rangle\right]+J(\langle\rho\rangle)=0,
$$

with the collision integral $J(\langle\rho\rangle)$ defined as

$$
J(\langle\rho\rangle)=\frac{i}{\hbar}\left\langle\left[U, g_{0}\right]\right\rangle .
$$

\section{B. Adding an electric field}

Let us now consider the effect of the driving electrostatic potential up to linear order. For simplicity we take this potential to have the form $V(\boldsymbol{r})=e \boldsymbol{E} \cdot \boldsymbol{r}$, implying a uniform electric field, which corresponds to the overwhelming majority of experimental setups. The case of inhomogeneous systems, including systems in inhomogeneous electric fields, entails additional subtleties which we postpone for later consideration [85-88]. Adding an electric field to the Hamiltonian implies a correction to the function $g$, which can then be written as $g=g_{0}+g_{E}$, where $g_{0}$ was found in the previous section, and

$$
\frac{\partial g_{E}}{\partial t}+\frac{i}{\hbar}\left[H_{0}, g_{E}\right]=-\frac{i}{\hbar}\left[V, g_{0}\right] .
$$

The notation $g_{E}$ reflects the fact that eventually it is the electric field that appears in the final expressions, rather than the electrostatic potential. For $g_{E}$ we find explicitly

$$
g_{E}=-\frac{i}{\hbar} \int_{0}^{\infty} d t^{\prime \prime} e^{-i H_{0} t^{\prime \prime} / \hbar}\left[V, g_{0}\left(t-t^{\prime \prime}\right)\right] e^{i H_{0} t^{\prime \prime} / \hbar} .
$$

The function $g_{E}$ is off diagonal in the momentum as well as in the band index. We solve Eq. (22) by introducing Markovian approximation which reads $g_{0}\left(t-t^{\prime \prime}\right) \approx g_{0}(t)$, whereupon in the frequency domain we obtain

$$
g_{E}=\frac{1}{2 \pi i} \int_{0}^{\infty} d \epsilon G_{0}^{R}(\epsilon)\left[V, g_{0}\right] G_{0}^{A}(\epsilon),
$$

and in the commutator, we should use Eq. (18) as a functional of the equilibrium distribution function $f_{0}(\epsilon)$ to fulfill the linear response.

The kinetic equation for the disorder-averaged density matrix $\langle\rho\rangle$ is now modified to

$$
\frac{\partial\langle\rho\rangle}{\partial t}+\frac{i}{\hbar}\left[H_{0},\langle\rho\rangle\right]+J_{0}(\langle\rho\rangle)=-\frac{i}{\hbar}[V,\langle\rho\rangle]-J_{E}(\langle\rho\rangle),
$$

with the collision integral $J_{E}(\langle\rho\rangle)$ defined as

$$
J_{E}(\langle\rho\rangle)=\frac{i}{\hbar}\left\langle\left[U, g_{E}\right]\right\rangle .
$$

Equation (24) is one of the main results of this work. In contrast to Luttinger's approach [62], we have taken the impurity average of the (reduced) density matrix first and reduced the quantum Liouville equation to a tractable kinetic equation for the disorder-averaged density matrix. Since the impurity average restores translational periodicity (in a noninteracting system), this enables us to work with only the $\boldsymbol{k}$-diagonal part of the density matrix, which significantly reduces the computational effort. The most important feature is the presence of the electric-field correction to the collision integral equation 
(25), which was not discussed in Ref. [50]. We note also that Eq. (23) was used in a different but equivalent form in a previous paper [89] in order to calculate side-jump effects in a system with extrinsic spin-orbit coupling. In this paper, we will focus on systems with intrinsic spin-obit coupling.

As we show below, the electric-field correction to the collision integral in Eq. (25) with the off-diagonal density function as given in Eq. (23) will provide results in agreement with previous calculations based on diagrammatic perturbation theory $[68,90]$. The Markovian approximation is indispensable here in order to obtain agreement with diagrammatic approaches. Specifically, Eq. (22) can be solved using two similar approximations. One is the Markovian approximation, which is tantamount to setting $g_{0}\left(t-t^{\prime \prime}\right) \rightarrow g_{0}(t)$; the alternative is to evolve $g_{0}$ in time from $t-t^{\prime \prime}$ to $t$ using the band Hamiltonian $H_{0}$ of the clean system. Only the Markovian approximation leads to exact agreement with diagrammatic results.

\section{Kinetic equation and linear response}

When Eq. (24) is expressed in the crystal momentum representation, we obtain the quantum kinetic equation [83]

$$
\frac{\partial f_{k}}{\partial t}+\frac{i}{\hbar}\left[H_{0 k}, f_{k}\right]+J_{0}\left(f_{k}\right)=\frac{e \boldsymbol{E}}{\hbar} \cdot \frac{D f_{k}}{D \boldsymbol{k}}-J_{E}\left(f_{k}\right) .
$$

We have written the matrix elements of $\langle\rho\rangle$ in this representation as $f_{k}$. We refer to $f_{k}$ henceforth as the density matrix, noting that it has matrix elements connecting different bands, although the band index $n$ has not been written explicitly. The covariant derivative $\frac{D f_{k}}{D k}=\frac{\partial f_{k}}{\partial k}-i\left[\mathcal{R}_{k}, f_{k}\right]$.

To solve Eq. (26), the density matrix is separated into a band-diagonal and a band-off-diagonal part; namely, we write $f_{k}=n_{k}+S_{k}$. The band-diagonal term $n_{k}$ represents the fraction of carriers in a specific band and is essentially the solution of the ordinary Boltzmann equation, while $S_{k}$ contains the effect of interband coherence, or band mixing. All our effort in recovering the semiclassical theory consists of eliminating $S_{k}$. The effective Boltzmann equation that we shall derive is simply what is obtained for $n_{k}$ once all references to $S_{k}$ have been eliminated. Fortunately, as we recapitulate below, the solution for $S_{k}$ in an electric field is relatively simple, making it straightforward to express expectation values in terms of $n_{k}$ alone.

The equilibrium density matrix is band diagonal, its elements represented by the Fermi-Dirac distribution for each band $n_{\mathrm{FD}}\left(\epsilon_{k}^{m}\right)$. In an electric field one may expand to linear order $f_{k}^{m n}=n_{\mathrm{FD}}\left(\epsilon_{k}^{m}\right) \delta_{m n}+f_{E k}^{m n}$, with corresponding expressions for $n_{E \boldsymbol{k}}$ and $S_{E \boldsymbol{k}}$. The kinetic equation is split into two coupled equations for $n_{E k}$ and $S_{E k}$, whose solution, based on an expansion in the small parameter $\hbar /\left(\epsilon_{F} \tau\right)$, is explained in detail in Ref. [50]. It was shown that $n_{E \boldsymbol{k}}$ starts at order -1 in this small parameter, since it is proportional to the scattering time $\tau$, while $S_{E k}$ starts at order 0 . Consequently, the subleading correction to $n_{E k}$, referred to as $n_{E k}^{(0)}$, is also required.

To leading order in $\hbar /\left(\epsilon_{F} \tau\right)$, the diagonal part reads

$$
\left[J_{0}\left(n_{E}^{(-1)}\right)\right]_{k}^{m}=\frac{e \boldsymbol{E}}{\hbar} \cdot \frac{\partial n_{\mathrm{FD}}\left(\epsilon_{\boldsymbol{k}}^{m}\right)}{\partial \boldsymbol{k}},
$$

where the Born approximation collision integral is

$$
\left[J_{0}\left(n_{E}\right)\right]_{k}^{m}=\frac{2 \pi}{\hbar} \sum_{m^{\prime}, \boldsymbol{k}^{\prime}}\left\langle U_{\boldsymbol{k} \boldsymbol{k}^{\prime}}^{m m^{\prime}} U_{\boldsymbol{k}^{\prime} \boldsymbol{k} \boldsymbol{k}}^{m^{\prime} m}\right\rangle\left(n_{E \boldsymbol{k}}^{m}-n_{E \boldsymbol{k}^{\prime}}^{m^{\prime}}\right) \delta\left(\epsilon_{\boldsymbol{k}}^{m}-\epsilon_{\boldsymbol{k}^{\prime}}^{m^{\prime}}\right) .
$$

The solution of Eq. (27) is in general rather complicated [91]. For a system with isotropic dispersion it reduces to the simple form

$$
n_{E \boldsymbol{k}}^{m(-1)}=\tau_{p}^{m} \frac{\boldsymbol{e}}{\hbar} \cdot \frac{\partial \epsilon_{\boldsymbol{k}}^{m}}{\partial \boldsymbol{k}} \frac{\partial n_{\mathrm{FD}}\left(\epsilon_{\boldsymbol{k}}^{m}\right)}{\partial \epsilon_{\boldsymbol{k}}^{m}},
$$

where the transport time $\tau_{p}^{m}$ is defined as

$$
\frac{1}{\tau_{p}^{m}}=\frac{2 \pi}{\hbar} \sum_{m^{\prime}, \boldsymbol{k}^{\prime}}\left\langle U_{\boldsymbol{k} \boldsymbol{k}^{\prime}}^{m m^{\prime}} U_{\boldsymbol{k}^{\prime} \boldsymbol{k}}^{m^{\prime} m}\right\rangle\left[1-\cos \left(\theta_{\boldsymbol{k}^{\prime}}-\theta_{\boldsymbol{k}}\right)\right] \delta\left(\epsilon_{\boldsymbol{k}}^{m}-\epsilon_{\boldsymbol{k}^{\prime}}^{m^{\prime}}\right) .
$$

The solution for $S_{E k}^{(0)}$ takes the simple form [50]

$$
S_{E \boldsymbol{k}}^{(0) m m^{\prime}}=\frac{\hbar\left(D+D^{\prime}\right)_{E \boldsymbol{k}}^{m m^{\prime}}}{i\left(\epsilon_{\boldsymbol{k}}^{m}-\epsilon_{\boldsymbol{k}}^{m^{\prime}}-i \eta\right)}
$$

with the intrinsic and anomalous driving terms [50]

$$
\begin{gathered}
D_{E \boldsymbol{k}}^{m m^{\prime}}=\frac{i e}{\hbar} \boldsymbol{E} \cdot \boldsymbol{\mathcal { R }}_{\boldsymbol{k}}^{m m^{\prime}}\left[n_{\mathrm{FD}}\left(\epsilon_{\boldsymbol{k}}^{m}\right)-n_{\mathrm{FD}}\left(\epsilon_{\boldsymbol{k}}^{m^{\prime}}\right)\right], \\
D_{E \boldsymbol{k}}^{\prime m m^{\prime}}=-\frac{\pi}{\hbar} \sum_{m^{\prime \prime}, \boldsymbol{k}^{\prime}}\left\langle U_{\boldsymbol{k} \boldsymbol{k}^{\prime}}^{m m^{\prime \prime}} U_{\boldsymbol{k}^{\prime} \boldsymbol{k}}^{m^{\prime \prime} m^{\prime}}\right\rangle\left\{\left(n_{E \boldsymbol{k}}^{m^{\prime}}-n_{E \boldsymbol{k}^{\prime}}^{m^{\prime \prime}} \delta\left(\epsilon_{m^{\prime} \boldsymbol{k}}-\epsilon_{m^{\prime \prime} \boldsymbol{k}^{\prime}}\right)+\left(n_{E \boldsymbol{k}}^{m}-n_{E \boldsymbol{k}^{\prime}}^{m^{\prime \prime}}\right) \delta\left(\epsilon_{m^{\prime \prime} \boldsymbol{k}^{\prime}}-\epsilon_{m \boldsymbol{k}}\right)\right\} .\right.
\end{gathered}
$$

Since $S_{E k}$ starts at zeroth order in the parameter $\hbar /\left(\epsilon_{F} \tau\right)$, we also require the subleading term $n_{E k}^{(0)}$, which is found from the equation

$$
\left[J_{0}\left(n_{E}^{(0)}\right)\right]_{k}^{m}=-\left[J_{s k}\left(n_{E}^{(-1)}\right)\right]_{k}^{m}-\left[J_{E}\left(n_{\mathrm{FD}}\right)\right]_{k}^{m},
$$

where the right-hand side acts as the driving term, whose constituents will be explained shortly. Solving this equation will yield two different contributions to the subleading diagonal density matrix $n_{E k}^{(0)}$, which we write as $n_{E k}^{(0)}=$ $n_{E \boldsymbol{k}}^{(\mathrm{sk})}+n_{E \boldsymbol{k}}^{(\mathrm{s})}$. Although both $n_{E \boldsymbol{k}}^{(\mathrm{sj})}$ and $n_{E \boldsymbol{k}}^{(\mathrm{sk})}$ are of zeroth order in $\hbar /\left(\epsilon_{F} \tau\right)$, they are parametrically different with respect to magnetization and Fermi energy, as we will see later on. We can solve for these two terms separately as follows.

The contribution $n_{E k}^{(\mathrm{sk})}$ stems from $D^{\prime}$ and is associated with skew scattering in the semiclassical theory. It is solved in an analogous manner to Eq. (27); namely, the driving term is found by substituting Eq. (31) into a collision integral of the form of Eq. (28), obtaining

$$
J_{0}\left[n_{E}^{(\mathrm{sk})}\right]=-\left[J_{s k}\left(n_{E}^{(-1)}\right)\right]_{k}^{m},
$$


which can be solved for $n_{E k}^{(\mathrm{sk})}$ using the standard techniques of Boltzmann theory [91]. The driving term in this equation can be written explicitly as a function of the leading-order density matrix $n_{E \boldsymbol{k}}^{(-1)}$ as

$$
\begin{aligned}
{\left[J_{s k}\left(n_{E}^{(-1)}\right)\right]_{k}^{m}=} & \frac{2 \pi^{2}}{\hbar} \sum_{m^{\prime} m^{\prime \prime} n \boldsymbol{k}^{\prime} \boldsymbol{k}^{\prime \prime}} \operatorname{Im}\left[\frac{\left\langle U_{\boldsymbol{k} \boldsymbol{k}^{\prime}}^{m m^{\prime \prime}} U_{\boldsymbol{k}^{\prime} \boldsymbol{k}}^{m^{\prime} m}\right\rangle\left\langle U_{\boldsymbol{k}^{\prime} \boldsymbol{k}^{\prime \prime}}^{m^{\prime \prime}} U_{\boldsymbol{k}^{\prime \prime} \boldsymbol{k}^{\prime}}^{n m^{\prime}}\right\rangle}{\left(\epsilon_{\boldsymbol{k}^{\prime}}^{m^{\prime \prime}}-\epsilon_{\boldsymbol{k}^{\prime}}^{m^{\prime}}\right)}\right]\left\{\left(n_{E \boldsymbol{k}^{\prime}}^{m^{\prime}(-1)}-n_{E \boldsymbol{k}^{\prime \prime}}^{n(-1)}\right) \delta\left(\epsilon_{\boldsymbol{k}^{\prime}}^{m^{\prime}}-\epsilon_{\boldsymbol{k}^{\prime \prime}}^{n}\right)+\left(n_{E \boldsymbol{k}^{\prime}}^{m^{\prime \prime}(-1)}-n_{E \boldsymbol{k}^{\prime \prime}}^{n(-1)}\right) \delta\left(\epsilon_{\boldsymbol{k}^{\prime \prime}}^{n}-\epsilon_{\boldsymbol{k}^{\prime}}^{m^{\prime \prime}}\right)\right\} \\
& \times \delta\left(\epsilon_{\boldsymbol{k}^{\prime}}^{m^{\prime \prime}}-\epsilon_{\boldsymbol{k}}^{m}\right)-\frac{2 \pi^{2}}{\hbar} \sum_{m^{\prime} m^{\prime \prime} n \boldsymbol{k}^{\prime} \boldsymbol{k}^{\prime \prime}} \operatorname{Im}\left[\frac{\left\langle U_{\boldsymbol{k} \boldsymbol{k}^{\prime}}^{m^{\prime \prime} m^{\prime}} U_{\boldsymbol{k}^{\prime} \boldsymbol{k}}^{m^{\prime} m}\right\rangle\left\langle U_{\boldsymbol{k} \boldsymbol{k}^{\prime \prime}}^{m n} U_{\boldsymbol{k}^{\prime \prime} \boldsymbol{k}}^{n m^{\prime \prime}}\right\rangle}{\left(\epsilon_{\boldsymbol{k}}^{m}-\epsilon_{\boldsymbol{k}}^{m^{\prime \prime}}\right)}\right] \\
& \times\left\{\left(n_{E \boldsymbol{k}}^{m^{\prime \prime}(-1)}-n_{E \boldsymbol{k}^{\prime \prime}}^{n(-1)}\right) \delta\left(\epsilon_{\boldsymbol{k}}^{m^{\prime \prime}}-\epsilon_{\boldsymbol{k}^{\prime \prime}}^{n}\right)+\left(n_{E \boldsymbol{k}}^{m(-1)}-n_{E \boldsymbol{k}^{\prime \prime}}^{n(-1)}\right) \delta\left(\epsilon_{\boldsymbol{k}^{\prime \prime}}^{n}-\epsilon_{\boldsymbol{k}}^{m}\right)\right\} \delta\left(\epsilon_{\boldsymbol{k}^{\prime}}^{m^{\prime}}-\epsilon_{\boldsymbol{k}}^{m^{\prime \prime}}\right),
\end{aligned}
$$

and we recall that $n_{E k}^{(-1)}$ was found in Eq. (29).

The second contribution to the driving term in Eq. (34) is due to the electric-field correction of the collision integral $J_{E}$ acting on the equilibrium distribution function. Since this contribution is associated with side-jump scattering in the semiclassical theory, we will refer to it as $n_{E \boldsymbol{k}}^{(\mathrm{sj})}$. To determine $n_{E \boldsymbol{k}}^{\text {(sj) }}$, we need to solve the equation

$$
J_{0}\left[n_{E}^{(\mathrm{sj})}\right]_{k}^{m}=-\left[J_{E}\left(n_{\mathrm{FD}}\right)\right]_{k}^{m} .
$$

In the crystal momentum representation the electric-field correction to the collision integral takes the form

$$
\begin{aligned}
{\left[J_{E}\left(n_{F D}\right)\right]_{\boldsymbol{k}}^{m} } & =\frac{2 \pi}{\hbar} \frac{\partial n_{F D}\left(\epsilon_{\boldsymbol{k}}^{m}\right)}{\partial \epsilon_{\boldsymbol{k}}^{m}} e \boldsymbol{E} \cdot \sum_{m^{\prime} \boldsymbol{k}^{\prime}}\left\langle U_{\boldsymbol{k} \boldsymbol{k}^{\prime}}^{m m^{\prime}} U_{\boldsymbol{k}^{\prime} \boldsymbol{k}}^{m^{\prime} m}\right\rangle\left[\boldsymbol{\mathcal { R }}_{\boldsymbol{k}^{\prime}}^{m^{\prime} m^{\prime}}-\boldsymbol{\mathcal { R }}_{\boldsymbol{k}}^{m m}\right] \delta\left(\epsilon_{\boldsymbol{k}^{\prime}}^{m^{\prime}}-\epsilon_{\boldsymbol{k}}^{m}\right) \\
& +\frac{2 \pi}{\hbar} \frac{\partial n_{F D}\left(\epsilon_{\boldsymbol{k}}^{m}\right)}{\partial \epsilon_{\boldsymbol{k}}^{m}} e \boldsymbol{E} \cdot \sum_{m^{\prime} \boldsymbol{k}^{\prime}} \operatorname{Im}\left\{\left\langle\left[\left(\nabla_{\boldsymbol{k}}+\nabla_{\boldsymbol{k}^{\prime}}\right) U_{\boldsymbol{k} \boldsymbol{k}^{\prime}}^{m m^{\prime}}\right] U_{\boldsymbol{k}^{\prime} \boldsymbol{k}}^{m^{\prime} m}\right\rangle\right\} \delta\left(\epsilon_{\boldsymbol{k}^{\prime}}^{m^{\prime}}-\epsilon_{\boldsymbol{k}}^{m}\right),
\end{aligned}
$$

where the derivatives act only on $U_{\boldsymbol{k} \boldsymbol{k}^{\prime}}^{m}$. This equation should be compared with the side-jump velocity calculated from a coordinate shift introduced in Ref. [81]. The balance between two collision integrals, as stated in Eq. (37), provides the necessary information to calculate a new subleading density function $n_{E}^{(\mathrm{sj})}$ that in the semiclassical language [92] is interpreted as an anomalous distribution due to coordinate shift of the scattered particle after many collisions.

\section{RECOVERING THE SEMICLASSICAL THEORY}

In this section we decompose the kinetic equation into a part representing carrier dynamics and a part representing the distribution, which is found from a modified Boltzmann equation. Since the equation of motion for the carrier wave vector, yielding $\hbar \dot{\boldsymbol{k}}=-e \boldsymbol{E}$, follows immediately from the operator commutator $[\boldsymbol{p}, V(\boldsymbol{r})]$, the bulk of our effort is devoted to finding the disorder-averaged velocity, which will yield the time evolution of the carrier position $\dot{\boldsymbol{r}}_{n}$. The prescription for recovering the semiclassical theory from the quantum kinetic equation proceeds as follows: (i) Determine the velocity expectation value as the operator trace $\operatorname{Tr}(\dot{\boldsymbol{r}} f)$, where $\dot{\boldsymbol{r}}=\frac{i}{\hbar}[H, \boldsymbol{r}]$ represents the matrix elements of the velocity operator. In the crystal momentum representation these are given by the covariant derivative $\dot{r}=$ $\frac{1}{\hbar} \frac{D H}{D k}$.

(ii) Reduce the trace to a form in which only band-diagonal elements of the density matrix appear. These will contain either the equilibrium Fermi-Dirac distribution $n_{0 k}$ or the correction to the band-diagonal part $n_{E k}$, which we recall has three constituents: $n_{E \boldsymbol{k}}=n_{E \boldsymbol{k}}^{(-1)}+n_{E \boldsymbol{k}}^{(s k)}+n_{E \boldsymbol{k}}^{(s j)}$.

(iii) The result follows a natural separation into a contribution associated with the equation of motion $\dot{\boldsymbol{r}}_{n}$ and one associated with the Boltzmann equation.

(iv) For the spin density, we follow similar steps; namely, we take the trace of the spin operator in the Bloch basis with the averaged density matrix. We will also find an extrinsic spin matrix element that accounts for spin rotations during scattering events.

The Hamiltonian is $H=H_{0}+V(\boldsymbol{r})+U(\boldsymbol{r})$, and since the last two terms commute with the position operator, they do not contribute to the velocity operator. The band Hamiltonian yields

$$
\begin{aligned}
\operatorname{Tr}(\dot{\boldsymbol{r}} f) \rightarrow \operatorname{Tr}\left\{\frac{i}{\hbar}\left[H_{0}, \boldsymbol{r}\right] f\right\} & =\frac{1}{\hbar} \sum_{m^{\prime}, m, \boldsymbol{k}}\left[\frac{\partial \epsilon_{\boldsymbol{k}}^{m^{\prime}}}{\partial \boldsymbol{k}} \delta_{m, m^{\prime}}+i\left(\epsilon_{\boldsymbol{k}}^{m}-\epsilon_{\boldsymbol{k}}^{m^{\prime}}\right) \boldsymbol{\mathcal { R }}_{\boldsymbol{k}}^{m m^{\prime}}\right] f_{\boldsymbol{k}}^{m^{\prime} m} \\
& =\sum_{m, \boldsymbol{k}} \boldsymbol{v}_{\boldsymbol{k}}^{m}\left[n_{E \boldsymbol{k}}^{(-1) m}+n_{\boldsymbol{E} \boldsymbol{k}}^{(0) m m}\right]+\frac{i}{\hbar} \sum_{m^{\prime}, m, \boldsymbol{k}}\left(\epsilon_{\boldsymbol{k}}^{m}-\epsilon_{\boldsymbol{k}}^{m^{\prime}}\right) \boldsymbol{\mathcal { R }}_{\boldsymbol{k}}^{m m^{\prime}} S_{E \boldsymbol{k}}^{(0) m^{\prime} m},
\end{aligned}
$$


with the Berry connection $\mathcal{R}_{\boldsymbol{k}}^{m m^{\prime}}=i\left\langle u_{k}^{m} \mid \nabla_{\boldsymbol{k}} u_{\boldsymbol{k}}^{m^{\prime}}\right\rangle$. The first term gives the usual group velocity $\boldsymbol{v}_{\boldsymbol{k}}^{m}=\nabla_{\boldsymbol{k}} \epsilon_{\boldsymbol{k}}^{m} / \hbar$, which is diagonal, while the second term gives a contribution due to band mixing and is purely off diagonal. We will concentrate on the second factor or band-mixing velocity. The off-diagonal density matrix is composed of two terms: an intrinsic one and an extrinsic one. Let us first consider the intrinsic one. It is

$$
\operatorname{Tr}\{\dot{\boldsymbol{r}} f\}^{\mathrm{int}}=-\sum_{m^{\prime}, m, \boldsymbol{k}} \mathcal{R}_{\boldsymbol{k}}^{m m^{\prime}} D_{E \boldsymbol{k}}^{m^{\prime} m} .
$$

After replacing the driving term $D_{E k}^{m^{\prime} m}$ by exchanging $m \rightarrow$ $m^{\prime}$ in the first term, and summing over intermediate states, the intrinsic contribution can be written as the average of the transverse velocity

$$
\operatorname{Tr}\{\dot{\boldsymbol{r}} f\}^{\mathrm{int}}=\frac{e}{\hbar} \sum_{m, \boldsymbol{k}} \boldsymbol{E} \times \boldsymbol{\Omega}_{\boldsymbol{k}}^{m} n_{\mathrm{FD}}\left(\epsilon_{\boldsymbol{k}}^{m}\right)
$$

with the Berry curvature

$$
\Omega_{k, z}^{m}=i\left[\left\langle\frac{\partial u_{k}^{m}}{\partial k_{x}} \mid \frac{\partial u_{k}^{m}}{\partial k_{y}}\right\rangle-\left\langle\frac{\partial u_{k}^{m}}{\partial k_{y}} \mid \frac{\partial u_{k}^{m}}{\partial k_{x}}\right\rangle\right] .
$$

The extrinsic contribution reads

$$
\operatorname{Tr}\{\dot{\boldsymbol{r}} f\}^{\mathrm{ext}}=\sum_{n, m, \boldsymbol{k}} \mathcal{R}_{\boldsymbol{k}}^{n m}[J(f)]_{k}^{m n} .
$$

After some algebra it can be written as

$$
\operatorname{Tr}\{\dot{\boldsymbol{r}} f\}^{\mathrm{ext}}=\langle\boldsymbol{\beta}\rangle=\sum_{m, \boldsymbol{k}} n_{E \boldsymbol{k}}^{(-1) m} \boldsymbol{\beta}_{\boldsymbol{k}}^{m},
$$

with the function $\boldsymbol{\beta}_{k}^{m}$ formally defined as

$$
\boldsymbol{\beta}_{k}^{m}=\frac{1}{2 \pi \hbar} \int_{-\infty}^{\infty} d \epsilon\left\langle\left[U, G_{0}^{A}(\epsilon)\left[U, \mathcal{R}^{\prime}\right] G_{0}^{R}(\epsilon)\right]\right\rangle_{k}^{m m},
$$

where the prime in $\mathcal{R}^{\prime}$ indicates that only the band-offdiagonal matrix elements of the Berry connection enter. In Eq. (45) we have written directly the electric-field-dependent correction to the distribution function, since Eq. (44) makes it obvious that this contribution vanishes when $f$ is replaced by the equilibrium distribution $n_{\mathrm{FD}}$. This is because, for scalar scattering as studied in this paper, the equilibrium distribution causes the entire collision integral to vanish. For computational evaluations it will be useful to list the explicit equation for $\boldsymbol{\beta}_{k}^{m}$ :

$\boldsymbol{\beta}_{\boldsymbol{k}}^{m}=\frac{\pi}{\hbar} \sum_{n, m^{\prime}, \boldsymbol{k}^{\prime}}\left\{\left[\mathcal{R}_{\boldsymbol{k}}^{\prime m n}\left\langle U_{\boldsymbol{k}, \boldsymbol{k}^{\prime}}^{n m^{\prime}} U_{\boldsymbol{k}^{\prime}, \boldsymbol{k}}^{m^{\prime} m}\right\rangle+\left\langle U_{\boldsymbol{k}, \boldsymbol{k}^{\prime}}^{m m^{\prime}} U_{\boldsymbol{k}^{\prime}, \boldsymbol{k}}^{m^{\prime} n}\right\rangle \boldsymbol{\mathcal { R }}_{\boldsymbol{k}}^{\prime n m}\right] \delta\left(\epsilon_{\boldsymbol{k}}^{m}-\epsilon_{\boldsymbol{k}^{\prime}}^{m^{\prime}}\right)-\left[\left\langle U_{\boldsymbol{k}, \boldsymbol{k}^{\prime}}^{m n} U_{\boldsymbol{k}^{\prime}, \boldsymbol{k}}^{m^{\prime} m}\right\rangle \mathcal{R}_{\boldsymbol{k}^{\prime}}^{\prime n m^{\prime}}+\left\langle U_{\boldsymbol{k}, \boldsymbol{k}^{\prime}}^{m m^{\prime}} U_{\boldsymbol{k}^{\prime}, \boldsymbol{k}}^{n m}\right\rangle \boldsymbol{\mathcal { R }}_{\boldsymbol{k}^{\prime}}^{\prime m^{\prime} n}\right] \delta\left(\epsilon_{\boldsymbol{k}^{\prime}}^{n}-\epsilon_{\boldsymbol{k}}^{m}\right)\right\}$.

Note that $\boldsymbol{\beta}_{k}^{m}$ is proportional to the disorder strength quantified here by $u_{0}^{2}$, making it first order in $\hbar /\left(\epsilon_{F} \tau\right)$. It represents a disorder-dependent correction to the semiclassical band velocity, which is independent of the applied electric field. Physically, $\boldsymbol{\beta}_{k}^{m}$ represents the average value of the random changes in the carrier velocity that occur every time the carrier is scattered between bands. Since $\boldsymbol{\beta}_{\boldsymbol{k}}^{m}$ has units of velocity and depends on the disorder potential, we will refer to it as the extrinsic velocity. Given that $\boldsymbol{\beta}_{k}^{m}$ is formally of first order in $\hbar /\left(\epsilon_{F} \tau\right)$, we are only interested in its product with the leading term in the distribution function, $n_{E k}^{(-1)}$, so that its overall contribution to the current is formally zeroth order in disorder. Moreover, with $n_{E k}^{(-1)}$ representing a Fermi surface contribution, the net effect of $\boldsymbol{\beta}_{\boldsymbol{k}}^{m}$ can be thought of as a random interband walk on the Fermi surface. Interestingly, $\boldsymbol{\beta}_{n}$ has the same mathematical form as the Born approximation scattering term $J_{0}$, except the band-off-diagonal elements of $\mathcal{R}$ appear instead of $n_{k}$. The presence of only the band-off-diagonal matrix elements of $\mathcal{R}$ ensures that $\boldsymbol{\beta}_{\boldsymbol{k}}^{m}$ is gauge covariant. In the examples we study below we find that $\boldsymbol{\beta}_{k}^{m}$ is nonzero in systems in which time-reversal symmetry is broken by, e.g., a magnetization. It is similar to the side jump appearing in Ref. [81], although we stress that our approach makes no reference to any coordinate shifts, the formal position operator is identical to the physical position operator, and the Berry curvature appearing in the semiclassical equations of motion below is the curvature for the clean system.

Philosophically, the approach we have adopted in this paper entails a change in one's point of view, according to which the semiclassical equations now describe carrier propagation averaged over many disorder scattering events. The carrier undergoes transitions between bands as it scatters, and its trajectory can be determined by averaging over impurity configurations.

Since all contributions to the current density are now expressed in terms of the distribution function (the equilibrium as well as the leading and subleading terms in an electric field), we are able to write the semiclassical equations of motion as

$$
\begin{gathered}
\dot{\boldsymbol{r}}_{m}=\frac{1}{\hbar} \frac{\partial \epsilon_{\boldsymbol{k}}^{m}}{\partial \boldsymbol{k}}-\dot{\boldsymbol{k}}_{m} \times \boldsymbol{\Omega}_{\boldsymbol{k}}^{m}+\boldsymbol{\beta}_{\boldsymbol{k}}^{m}, \\
\hbar \dot{\boldsymbol{k}}_{m}=-e \boldsymbol{E} .
\end{gathered}
$$

The distribution function is found from the Boltzmann equation, with the caveat that we require both the leadingand subleading-order terms in the disorder strength. The procedure is as follows. First the leading-order term in the distribution function $n_{E k}^{(-1)}$ is found from

$$
J_{0}\left[n_{E}^{(-1)}\right]_{k}^{m}=\frac{e \boldsymbol{E}}{\hbar} \cdot \frac{\partial n_{\mathrm{FD}}\left(\epsilon_{\boldsymbol{k}}^{m}\right)}{\partial \boldsymbol{k}},
$$

while the subleading correction $n_{E}^{(0)}$ is given by

$$
J_{0}\left[n_{E}^{(0)}\right]_{k}^{m}=-\left[J_{E}\left(n_{\mathrm{FD}}\right)\right]_{k}^{m}-J_{s k}\left[n_{E}^{(-1)}\right]_{k}^{m},
$$


where the left-hand side is the quantity to be found and the right-hand side plays the role of a driving term. Finally, we are able to write the full expectation value of the current in terms of semiclassical quantities

$$
\langle\boldsymbol{j}\rangle=(-e) \sum_{m \boldsymbol{k}}\left\{\frac{1}{\hbar} \frac{\partial \epsilon_{\boldsymbol{k}}^{m}}{\partial \boldsymbol{k}}\left[n_{E \boldsymbol{k}}^{(-1) m}+n_{E \boldsymbol{k}}^{(s k) m}+n_{E \boldsymbol{k}}^{(s j) m}\right]+\left(\frac{e \boldsymbol{E}}{\hbar} \times \boldsymbol{\Omega}_{\boldsymbol{k}}^{m}\right) n_{\mathrm{FD}}\left(\epsilon_{\boldsymbol{k}}^{m}\right)+\boldsymbol{\beta}_{\boldsymbol{k}}^{m} n_{E \boldsymbol{k}}^{(-1) m}\right\} .
$$

Thus, in order to recover the quantum mechanical result for coherent scattering off an external potential, one needs to consider disorder-induced changes both in the distribution function, up to order zero in the disorder potential, and in the carrier dynamics, in the form of an extrinsic velocity. We have shown that disorder affects not only the state occupation but also the semiclassical equations of motion, generating a correction to the velocity that accounts for band mixing mediated by disorder, in addition to the intrinsic band-mixing effects mediated by the Berry curvature. Referring to Sec. III and recalling that the semiclassical theory was derived by eliminating $S_{k}$, we note that the coupling between $n_{k}$ and $S_{k}$ represents spin-charge coupling, which essentially involves a feedback effect of spin dynamics onto charge dynamics. This is to be expected in spin-orbit coupled systems, to which our theory finds a direct application: On the one hand, as the particle accelerates, its wave vector changes, bringing with it a spin rotation; on the other hand, spin rotations in turn cause changes in the wave vector and therefore in the trajectory of the particle. In the future, by adding spatial dependence explicitly, the full equations describing coupled spin and charge diffusion can be derived, in analogy with Ref. [80]. We emphasize, finally, that since the denominator in $S_{\boldsymbol{k}}$ involves the energy difference between bands, the bands are assumed to be nondegenerate (although the energy difference can be very small). The case of fully degenerate bands requires further development.

Let us consider the expectation value of the spin operator in the presence of an electric field

$$
\operatorname{Tr}\{\boldsymbol{s} f\}=\sum_{m, \boldsymbol{k}} \boldsymbol{s}_{\boldsymbol{k}}^{m m} n_{E \boldsymbol{k}}^{m}+\sum_{n, m, \boldsymbol{k}} \boldsymbol{s}_{\boldsymbol{k}}^{n m} S_{E \boldsymbol{k}}^{m n},
$$

where $\boldsymbol{s}_{\boldsymbol{k}}^{n m}$ represent the matrix elements of the spin operator. Writing explicitly the off-diagonal terms of the density matrix in the average of the spin operator, we can separate the intrinsic and extrinsic contributions as

$$
\begin{aligned}
\langle\boldsymbol{s}\rangle^{\mathrm{int}} & =\sum_{n, m, \boldsymbol{k}} \frac{\hbar \boldsymbol{s}_{n m}}{i\left(\epsilon_{\boldsymbol{k}}^{m}-\epsilon_{\boldsymbol{k}}^{n}\right)} D_{E \boldsymbol{k}}^{m n}, \\
\langle\boldsymbol{s}\rangle^{\mathrm{ext}} & =\sum_{n, m, \boldsymbol{k}} \frac{\hbar \boldsymbol{s}_{\boldsymbol{k}}^{n m}}{i\left(\epsilon_{\boldsymbol{k}}^{m}-\epsilon_{\boldsymbol{k}}^{n}\right)} D_{E \boldsymbol{k}}^{\prime m n} .
\end{aligned}
$$

If we define the quantity

$$
\mathcal{T}_{\boldsymbol{k}}^{n m}=\frac{\hbar}{i\left(\epsilon_{\boldsymbol{k}}^{n}-\epsilon_{\boldsymbol{k}}^{m}\right)} \boldsymbol{s}_{\boldsymbol{k}}^{n m}
$$

the intrinsic contribution can be rewritten as

$$
\langle\boldsymbol{s}\rangle^{\mathrm{int}}=\frac{i e}{\hbar} \sum_{m, \boldsymbol{k}}\left[\mathcal{T}_{\boldsymbol{k}}, \boldsymbol{E} \cdot \boldsymbol{\mathcal { R }}_{\boldsymbol{k}}^{\prime}\right]^{m} n_{\mathrm{FD}}\left(\varepsilon_{\boldsymbol{k}}^{m}\right),
$$

where we only have to take off-diagonal components inside the commutator. This enables us to define an intrinsic spin expectation value as

$$
\boldsymbol{s}_{\boldsymbol{k}}^{\mathrm{int}}=\frac{i e}{\hbar}\left[\mathcal{T}_{\boldsymbol{k}}, \boldsymbol{E} \cdot \boldsymbol{\mathcal { R }}_{\boldsymbol{k}}^{\prime}\right]^{m}
$$

The extrinsic part can be written as

$$
\langle\boldsymbol{s}\rangle^{\mathrm{ext}}=\sum_{n, m, \boldsymbol{k}} \mathcal{T}_{\boldsymbol{k}}^{n m}\left[J_{0}\left(n_{E}^{(-1)}\right)\right]_{\boldsymbol{k}}^{m n}
$$

This is mathematically analogous to the expression for the extrinsic velocity equation (44), and using a similar manipulation, we can reexpress it as

$$
\langle\boldsymbol{s}\rangle_{\mathrm{bm}}^{\mathrm{ext}}=\sum_{m, \boldsymbol{k}} n_{E \boldsymbol{k}}^{(-1) m} \boldsymbol{\gamma}_{\boldsymbol{k}}^{m m},
$$

where $n_{E k}^{(-1)}$ is the leading-order distribution function and we introduce the new extrinsic spin $\boldsymbol{\gamma}_{\boldsymbol{k}}^{m}$ given by

$$
\gamma_{k}^{m}=\frac{1}{2 \pi \hbar} \int_{-\infty}^{\infty} d \epsilon\left\langle\left[U, G_{0}^{A}(\epsilon)[U, \mathcal{T}] G_{0}^{R}(\epsilon)\right]\right\rangle_{k}^{m m}
$$

In exact analogy with $\boldsymbol{\beta}_{\boldsymbol{k}}^{m}$, since the Born approximation scattering term vanishes when the distribution function is replaced by the Fermi-Dirac distribution, $\boldsymbol{\gamma}_{\boldsymbol{k}}^{m}$ also vanishes in equilibrium in the presence of scalar scattering. This quantity represents spin rotations during scattering events. Again, the quantity $\mathcal{T}$ only has interband matrix elements. Explicitly, $\boldsymbol{\gamma}_{\boldsymbol{k}}^{m}$ is evaluated as

$$
\begin{aligned}
\boldsymbol{\gamma}_{\boldsymbol{k}}^{m m}= & \frac{\pi}{\hbar} \sum_{n, m^{\prime}, \boldsymbol{k}^{\prime}}\left\{\left[\mathcal{T}_{\boldsymbol{k}}^{m, n}\left\langle U_{\boldsymbol{k}, \boldsymbol{k}^{\prime}}^{n m^{\prime}} U_{\boldsymbol{k}^{\prime}, \boldsymbol{k}}^{m^{\prime} m}\right\rangle+\left\langle U_{\boldsymbol{k}, \boldsymbol{k}^{\prime}}^{m m^{\prime}} U_{\boldsymbol{k}^{\prime}, \boldsymbol{k}}^{m^{\prime} n}\right\rangle \mathcal{T}_{\boldsymbol{k}}^{n, m}\right] \delta\left(\epsilon_{\boldsymbol{k}}^{m}-\epsilon_{\boldsymbol{k}^{\prime}}^{m^{\prime}}\right)\right. \\
& \left.-\left[\left\langle U_{\boldsymbol{k}, \boldsymbol{k}^{\prime}}^{m n} U_{\boldsymbol{k}^{\prime}, \boldsymbol{k}}^{m^{\prime} m}\right\rangle \mathcal{T}_{\boldsymbol{k}^{\prime}}^{n, m^{\prime}}+\left\langle U_{\boldsymbol{k}, \boldsymbol{k}^{\prime}}^{m m^{\prime}} U_{\boldsymbol{k}^{\prime}, \boldsymbol{k}}^{n m}\right\rangle \mathcal{T}_{\boldsymbol{k}^{\prime}}^{m^{\prime}, n}\right] \delta\left(\epsilon_{\boldsymbol{k}^{\prime}}^{n}-\epsilon_{\boldsymbol{k}}^{m}\right)\right\} .
\end{aligned}
$$


In analogy to the above, we can write a modified intraband spin matrix element as

$$
\boldsymbol{s}^{m m} \rightarrow \boldsymbol{s}_{\boldsymbol{k}}^{m m}+\frac{i e}{\hbar}\left[\mathcal{T}_{\boldsymbol{k}}, \boldsymbol{E} \cdot \boldsymbol{\mathcal { R }}_{\boldsymbol{k}}^{\prime}\right]^{m}+\boldsymbol{\gamma}_{\boldsymbol{k}}^{m} .
$$

The first factor is the bare matrix element of the spin operator in band $m$, while the remaining two represent nonequilibrium corrections, the first being intrinsic and the second extrinsic. We note that $\boldsymbol{\gamma}_{\boldsymbol{k}}$ and $\boldsymbol{\beta}_{\boldsymbol{k}}$ are mathematically very similar and both contribute at the Fermi energy. The intrinsic contribution to the spin density from the Fermi sea appears in the second term in Eq. (62). The full semiclassical expression for the spin density is given by

$$
\langle\boldsymbol{s}\rangle=\sum_{m \boldsymbol{k}}\left\{\boldsymbol{s}_{\boldsymbol{k}}^{m}\left[n_{\mathrm{FD}}\left(\epsilon_{\boldsymbol{k}}^{m}\right)+n_{E \boldsymbol{k}}^{(-1) m}+n_{E \boldsymbol{k}}^{(s k) m}+n_{E \boldsymbol{k}}^{(s j) m}\right]+\frac{i e}{\hbar}\left[\mathcal{T}_{\boldsymbol{k}}, \boldsymbol{E} \cdot \boldsymbol{\mathcal { R }}_{\boldsymbol{k}}^{\prime}\right]^{m} n_{\mathrm{FD}}\left(\epsilon_{\boldsymbol{k}}^{m}\right)+\boldsymbol{\gamma}_{\boldsymbol{k}}^{m} n_{E \boldsymbol{k}}^{(-1) m}\right\} .
$$

\section{LINEAR RESPONSE FAMILY TREE}

In closing the methodological discussion we remark briefly on the relationships between the various linear response theories. The most common strategy for solving Eq. (11) in an electric field is via Kubo linear response theory, which is discussed in detail in many textbooks [93]; hence we only dwell upon its fundamental aspects. Briefly, the Hamiltonian is decomposed as $H=\left(H_{0}+U\right)+V$, and the nonequilibrium part of the density matrix is likewise singled out as $\rho=\rho_{0}+\rho_{E}$. Then in linear response one can write

$$
\frac{\partial \rho_{E}}{\partial t}+\frac{i}{\hbar}\left[H_{0}+U, \rho_{E}\right]=-\frac{i}{\hbar}\left[V, \rho_{0}\right] .
$$

This equation is solved immediately to yield

$$
\rho_{E}=\frac{1}{2 \pi i} \int_{0}^{\infty} d \epsilon\left[G^{R}(\epsilon) V G^{A}(\epsilon), \rho_{0}\right],
$$

where $G^{R}, G^{A}$ are the retarded and advanced Green's functions, respectively, for the disordered system

$$
G^{R}(\epsilon)=-\frac{i}{\hbar} \int_{0}^{\infty} d t e^{-i\left(H_{0}+U\right) t / \hbar} e^{i \epsilon t / \hbar} e^{-\eta t}
$$

We refrain from writing out the energy dependence in full. Note that the Green's functions have not been averaged over disorder configurations at this stage. To obtain the customary Kubo formula, one must trace over the velocity operator and average over impurity configurations

$$
\langle\boldsymbol{j}\rangle=-\frac{e}{2 \pi i} \int_{0}^{\infty} d \epsilon \operatorname{tr}\left\langle\boldsymbol{v}\left[G^{R} V G^{A}, \rho_{0}\right]\right\rangle .
$$

The procedure is standard, so we do not cover it here in detail; the purpose of this description is illustrative. The important point to notice is that the Kubo formula is the integral approach to solving the Liouville equation, whereas the kinetic equation we follow in this paper represents the differential approach, or integrodifferential in view of the complex scattering term. The Keldysh theory follows a similar path, and although it takes as its starting point a series of Green's functions, its ultimate origin lies in the quantum Liouville equation. The Keldysh theory is formally nonlocal in time, although in the vast majority of practical applications the nonlocality is removed and the Keldysh Green's function, which is analogous to the density matrix employed in this paper, depends only on the difference in time variables. Thus, for the purposes of the present comparison, the quantum Boltzmann equation derived in the Keldysh theory is indistinguishable from the quantum kinetic equation derived from the density matrix. The Kubo formula, Keldysh theory, and quantum kinetic equation may be regarded as holistic approaches, in which both the carrier dynamics and the carrier distribution are accounted for in the density matrix (or Keldysh Green's function), and the net result is the expectation value of a physical observable. In contrast, the semiclassical theory involves a separation between the carrier dynamics and carrier distribution, which can help to build an intuitive picture of the underlying physics. The relationship between the different approaches is summarized in the family tree of Fig. 1. The most important observation in this context is the common origin of all linear response theories, which reinforces the expectation that they should all lead to the same results.

\section{APPLICATIONS}

We now turn to applications of the theory, which are intended to illustrate the way the extrinsic velocity, extrinsic spin terms, and additional scattering terms in the Boltzmann equation appear in the explicit evaluations of physical observables for model systems. In particular, we emphasize the relationship between these various contributions and the analogous quantities appearing in diagrammatic theories, which enables us to reconcile the semiclassical and diagrammatic results. Our focus will be on topological insulators [94], where we discuss the anomalous Hall effect as well as spin-orbit torques, and compare the semiclassical results with previous work.

\section{A. Anomalous Hall effect in topological insulators}

In this section we calculate the anomalous Hall conductivity in topological insulators. The anomalous Hall conductivity

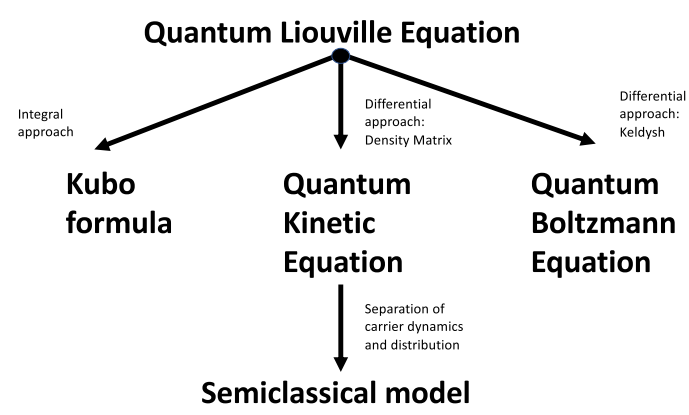

FIG. 1. Family tree of linear response theories. 
is basically expressed in terms of four contributions: an intrinsic contribution that takes into account the whole Fermi sea of the system, an extrinsic contribution due to the extrinsic velocity $\boldsymbol{\beta}_{\boldsymbol{k}}^{m}$ at the Fermi surface, a side-jump-like contribution at the Fermi energy due to an electric-field correction to the collision integral, and a skew scattering contribution.

The Hamiltonian that describes low-energy excitations in the surface of three-dimensional (3D) topological insulators reads

$$
H=\hbar v_{F}\left(k_{x} \sigma_{y}-k_{y} \sigma_{x}\right)+M \sigma_{z},
$$

where $v_{F}$ is the effective Fermi velocity and $\sigma_{i}$ are Pauli matrices, while $M$ is the magnetization. The eigenvalues are $\epsilon_{\boldsymbol{k}}^{ \pm}= \pm \sqrt{\hbar^{2} v_{F}^{2} k^{2}+M^{2}}$, where \pm labels the conduction or valence band, and the eigenstates are

$$
\left|u_{k}^{ \pm}\right\rangle=\frac{1}{\sqrt{2}}\left(\begin{array}{c}
e^{-i \theta_{k}} \sqrt{1 \pm \xi_{k}} \\
\pm i \sqrt{1 \mp \xi_{k}}
\end{array}\right),
$$

with a parameter $\xi_{k}=M / \lambda_{k}$ with $\lambda_{k}=\sqrt{\hbar^{2} v_{F}^{2} k^{2}+M^{2}}$ and $\theta_{\boldsymbol{k}}=\arctan \left(k_{y} / k_{x}\right)$.

From the eigenstates we determine the Berry connection vector $\mathcal{R}_{\boldsymbol{k}}^{m m^{\prime}}=i\left\langle u_{\boldsymbol{k}}^{m} \mid \nabla_{\boldsymbol{k}} u_{\boldsymbol{k}}^{m^{\prime}}\right\rangle$. We decompose this vector into a diagonal and an off-diagonal contribution, namely, $\boldsymbol{\mathcal { R }}_{\boldsymbol{k}}=$ $\boldsymbol{\mathcal { R }}_{k}^{d}+\boldsymbol{\mathcal { R }}_{k}^{\text {od }}$, with

$$
\begin{aligned}
\mathcal{R}_{\boldsymbol{k}}^{d} & =\frac{\hat{\boldsymbol{\theta}}}{2 k}\left(\sigma_{0}+\xi_{\boldsymbol{k}} \sigma_{z}\right), \\
\mathcal{R}_{\boldsymbol{k}}^{o d} & =\frac{\hbar v_{F}}{2 \lambda_{\boldsymbol{k}}}\left(\sigma_{x} \hat{\boldsymbol{\theta}}-\xi_{\boldsymbol{k}} \sigma_{y} \hat{\boldsymbol{k}}\right) .
\end{aligned}
$$

We have defined a unit vector parallel to momentum $\hat{\boldsymbol{k}}=$ $\left(\cos \theta_{\boldsymbol{k}}, \sin \theta_{\boldsymbol{k}}\right)$ and a unit vector perpendicular to momentum $\hat{\boldsymbol{\theta}}=\left(-\sin \theta_{\boldsymbol{k}}, \cos \theta_{\boldsymbol{k}}\right)$. Also, these vectors are related as $\hat{\boldsymbol{\theta}}=$ $\hat{z} \times \hat{\boldsymbol{k}}$.

Let us start by calculating the intrinsic contribution to the Hall conductivity. It reads

$$
\sigma_{y x}^{\mathrm{int}}=\frac{e^{2}}{\hbar} \sum_{m, \boldsymbol{k}} \Omega_{\boldsymbol{k}, z}^{m} n_{\mathrm{FD}}\left(\epsilon_{\boldsymbol{k}}^{m}\right) .
$$

Explicit evaluation for a topological insulator gives the Berry curvature

$$
\Omega_{k, z}^{ \pm}=\mp \frac{\xi_{k}\left(1-\xi_{k}^{2}\right)}{2 k^{2}}
$$

After a straightforward integration we find the intrinsic contribution to the Hall conductivity to be

$$
\sigma_{y x}^{\text {int }}=\frac{e^{2}}{4 \pi \hbar} \frac{M}{\epsilon_{F}} .
$$

The extrinsic velocity makes the following contribution to the anomalous Hall conductivity:

$$
\sigma_{y x}^{\mathrm{ext}}=-\frac{e}{E_{x}} \sum_{m, \boldsymbol{k}} n_{E \boldsymbol{k}}^{(-1) m} \beta_{\boldsymbol{k}, y}^{m} .
$$

The leading-order correction to the distribution function is $n_{E \boldsymbol{k}}^{(-1) m}=-e \tau_{t r} \boldsymbol{E} \cdot \boldsymbol{v}_{\boldsymbol{k}}^{m} \delta\left(\epsilon_{\boldsymbol{k}}^{m}-\epsilon_{F}\right)$ with the transport time given by the expression

$$
\frac{1}{\tau_{t r}}=\frac{1}{2 \tau}\left(1+3 \xi_{k}^{2}\right),
$$

with the scattering time defined as $1 / \tau=\pi u_{0}^{2} \rho\left(\epsilon_{\boldsymbol{k}}\right) / \hbar$ and the density of states $\rho\left(\epsilon_{k}\right)=\lambda_{k} / 2 \pi \hbar^{2} v_{F}^{2}$. The diagonal velocity is $v_{x}=v_{F}\left(1-\xi_{k}^{2}\right)^{1 / 2} \cos \theta_{k}$, and the extrinsic velocity in the conduction band takes the form

$$
\boldsymbol{\beta}=\sigma_{0} \frac{1}{\tau} \frac{\hbar v_{F}}{\lambda_{k}} \xi_{k}\left(1-\xi_{k}^{2}\right)^{1 / 2} \hat{\boldsymbol{\theta}} .
$$

The extrinsic velocity is a transverse velocity since it is proportional to the unit vector $\hat{\boldsymbol{\theta}}$. After explicit integration we arrive at the expression

$$
\sigma_{y x}^{\mathrm{ext}}=\frac{e^{2}}{2 \pi \hbar} \frac{M}{\epsilon_{F}} \frac{\left(1-\xi_{F}^{2}\right)}{\left(1+3 \xi_{F}^{2}\right)} .
$$

Notice that $n_{E \boldsymbol{k}}^{(-1) m}$ is inversely proportional to the impurity density, while the extrinsic velocity $\beta$ is proportional to the impurity density. As a result, the overall effect in Eq. (75) is independent of disorder. The extrinsic velocity $\boldsymbol{\beta}_{\boldsymbol{k}}^{m}$ comprises the effect of disorder on carrier dynamics, namely, it can be interpreted as an effective velocity of the electron after many collisions, in contrast to the group velocity, which is a velocity between collisions. In this sense our extrinsic velocity can be associated with the side-jump velocity encountered in previous semiclassical results [92] but with the difference that $\boldsymbol{\beta}_{\boldsymbol{k}}^{m}$ is entirely due to band mixing mediated by the off-diagonal components of the Berry connection vector and that it is constructed from a collision integral without introducing any quantity related to coordinate shift.

As we discussed earlier, there are also two contributions to the anomalous Hall conductivity related to two different diagonal subleading density matrix functions. Let us first calculate the anomalous Hall conductivity related to the term $n_{E \boldsymbol{k}}^{(\mathrm{sj})}$ in the distribution function. It reads

$$
\sigma_{y x}^{\mathrm{sj}}=-\frac{e}{E_{x}} \sum_{\boldsymbol{k}} v_{\boldsymbol{k}, y}^{++} n_{E \boldsymbol{k}}^{(\mathrm{sj})++} .
$$

The diagonal velocity reads

$$
v_{k, y}^{++}=v_{F}\left(1-\xi_{k}^{2}\right)^{1 / 2} \sin \theta_{k},
$$

while the correction to the distribution function is

$$
n_{E \boldsymbol{k}}^{(\mathrm{s})++}=2 \frac{\hbar v_{F}}{\lambda_{k}} \xi_{k} \delta\left(\epsilon_{F}-\epsilon_{\boldsymbol{k}}^{+}\right) \frac{\left(1-\xi_{k}^{2}\right)^{1 / 2}}{\left(1+3 \xi_{\boldsymbol{k}}^{2}\right)} e \boldsymbol{E} \cdot \hat{\boldsymbol{\theta}} .
$$

Replacing all elements, we find for the conductivity

$$
\sigma_{y x}^{\mathrm{sj}}=\frac{e^{2}}{2 \pi \hbar} \frac{M}{\epsilon_{F}} \frac{\left(1-\xi_{F}^{2}\right)}{\left(1+3 \xi_{F}^{2}\right)} .
$$

This term doubles the contribution in Eq. (78) due to the extrinsic velocity, although in this case the effect of disorder is completely captured by $n_{E k}^{(\mathrm{sj})}$.

In previous semiclassical studies an anomalous distribution function was introduced as a result of a coordinate shift [92]. In contrast, we have derived $n_{E \boldsymbol{k}}^{\text {(sj) }}$ from Eq. (37) without any need for introducing a coordinate shift. 
The contribution to the anomalous Hall conductivity related to the skew scattering correction to the distribution function, $n_{E \boldsymbol{k}}^{(\mathrm{sk})}$, reads

$$
\sigma_{y x}^{\mathrm{sk}}=-\frac{e}{E_{x}} \sum_{\boldsymbol{k}}\left[v_{\boldsymbol{k}, y}^{++} n_{E \boldsymbol{k}}^{(\mathrm{sk})++}+v_{\boldsymbol{k}, y}^{--} n_{E \boldsymbol{k}}^{(\mathrm{sk})--}\right] .
$$

The diagonal velocities are $v_{k, y}^{ \pm \pm}= \pm v_{F}\left(1-\xi_{k}^{2}\right)^{1 / 2} \sin \theta_{k}$, and

$$
n_{E \boldsymbol{k}}^{(\mathrm{sk})}=\frac{3}{2} \frac{\hbar v_{F}}{\lambda_{k}} \xi_{k} \frac{\left(1-\xi_{F}^{2}\right)^{3 / 2}}{\left(1+3 \xi_{F}^{2}\right)^{2}} \delta\left(\epsilon_{F}-\epsilon_{\boldsymbol{k}}^{+}\right) e \boldsymbol{E} \cdot \hat{\boldsymbol{\theta}} \sigma_{z} .
$$

After integration we obtain

$$
\sigma_{y x}^{\mathrm{sk}}=\frac{e^{2}}{2 \pi \hbar} \frac{M}{\epsilon_{F}} \frac{3\left(1-\xi_{F}^{2}\right)^{2}}{2\left(1+3 \xi_{F}^{2}\right)^{2}} .
$$

Adding all the contributions to the Hall conductivity, we get the final expression

$$
\sigma_{y x}=\frac{4 e^{2}}{2 \pi \hbar} \frac{M}{\epsilon_{F}}\left[\frac{1+\xi_{F}^{2}}{\left(1+3 \xi_{F}^{2}\right)^{2}}\right],
$$

in exact agreement with previous results using the noncrossing approximation and diagrammatic perturbation theory $[68,75,76,82,92,95]$. We note that in Ref. [96] the term $J_{E}$ was not evaluated explicitly for the scalar part of the scattering potential. When this is done, the result agrees with Eq. (86).

\section{B. Spin density and spin-orbit torques in topological insulators}

In this section we determine the spin density and spin-orbit torques in topological insulators with an out-of-plane magnetization described by the effective Hamiltonian equation (68). As for the conductivity, the spin density has five contributions: a dominant contribution from the Fermi surface leading to the Edelstein effect [97], an intrinsic contribution from the Fermi sea, a contribution due to the extrinsic spin expectation value $\gamma_{k}^{m}$ at the Fermi surface, a side-jump-like contribution at the Fermi energy due to the electric-field correction to the collision integral, and a skew scattering contribution.

The leading-order contribution to the spin density is

$$
\langle\boldsymbol{s}\rangle^{\text {Edel }}=\sum_{m, \boldsymbol{k}} \boldsymbol{s}_{\boldsymbol{k}}^{m m} n_{E \boldsymbol{k}}^{(-1) m} .
$$

Using $n_{E \boldsymbol{k}}^{(-1) m}=-e \tau_{t r} \boldsymbol{E} \cdot \boldsymbol{v}_{\boldsymbol{k}}^{m} \delta\left(\epsilon_{\boldsymbol{k}}^{m}-\epsilon_{F}\right)$ and

$$
\begin{aligned}
s_{k, x}^{ \pm \pm} & =\mp\left(1-\xi_{k}^{2}\right)^{1 / 2} \sin \theta_{k}, \\
s_{k, y}^{ \pm \pm} & = \pm\left(1-\xi_{k}^{2}\right)^{1 / 2} \cos \theta_{k},
\end{aligned}
$$

the Edelstein effect contribution to the spin density is

$$
\langle\boldsymbol{s}\rangle^{\mathrm{Edel}}=e \tau \boldsymbol{E} \times \hat{z} v_{F} \rho\left(\epsilon_{F}\right) \frac{1-\xi_{F}^{2}}{1+3 \xi_{F}^{2}} .
$$

The fraction of the spin density weighted by the intrinsic driving term reads

$$
\langle\boldsymbol{s}\rangle^{\mathrm{int}}=\frac{i e \boldsymbol{E}}{\hbar} \cdot \sum_{n m \boldsymbol{k}}\left(\mathcal{T}_{\boldsymbol{k}}^{m n} \boldsymbol{\mathcal { R }}_{\boldsymbol{k}}^{n m}-\mathcal{R}_{\boldsymbol{k}}^{m n} \mathcal{T}_{\boldsymbol{k}}^{n m}\right) n_{\mathrm{FD}}\left(\varepsilon_{\boldsymbol{k}}^{m}\right) .
$$

The dot product is between the electric field and the Berry connection vector. With the Berry connection

$$
\begin{gathered}
\mathcal{R}_{\boldsymbol{k}, x}^{+-}=-\frac{\hbar v_{F}}{2 \lambda_{k}}\left(\sin \theta_{\boldsymbol{k}}-i \xi_{\boldsymbol{k}} \cos \theta_{\boldsymbol{k}}\right), \\
\mathcal{R}_{\boldsymbol{k}, y}^{+-}=\frac{\hbar v_{F}}{2 \lambda}\left(\cos \theta_{\boldsymbol{k}}+i \xi_{\boldsymbol{k}} \sin \theta_{\boldsymbol{k}}\right)
\end{gathered}
$$

and the off-diagonal spin expectation values

$$
\begin{aligned}
s_{k, x}^{-+} & =\left(\xi_{k} \sin \theta+i \cos \theta\right), \\
s_{k, y}^{-+} & =-\left(\xi_{k} \cos \theta-i \sin \theta\right)
\end{aligned}
$$

we get the intrinsic correction to the spin density

$$
\langle\boldsymbol{s}\rangle^{\text {int }}=\frac{e M \hbar v_{F} \rho\left(\epsilon_{F}\right)}{2 \epsilon_{F}^{2}} \boldsymbol{E} .
$$

The extrinsic correction is defined as

$$
\begin{gathered}
\langle\boldsymbol{s}\rangle^{\mathrm{ext}}=\sum_{m, \boldsymbol{k}} n_{E \boldsymbol{k}}^{(-1) m} \boldsymbol{\gamma}_{\boldsymbol{k}}^{m}, \\
\boldsymbol{\gamma}_{\boldsymbol{k}}^{++}=-\sigma_{0} \frac{1}{\tau} \frac{\hbar}{\lambda_{\boldsymbol{k}}} \xi_{k}\left(1-\xi_{k}^{2}\right)^{1 / 2} \hat{\boldsymbol{k}},
\end{gathered}
$$

yielding

$$
\langle\boldsymbol{s}\rangle^{\mathrm{ext}}=\frac{e \hbar v_{F} \rho\left(\epsilon_{F}\right) M}{\epsilon_{F}^{2}} \frac{1-\xi_{F}^{2}}{1+3 \xi_{F}^{2}} \boldsymbol{E} .
$$

This extrinsic contribution to the spin density is the counterpart of the extrinsic velocity contribution in the anomalous Hall effect. The extrinsic spin $\boldsymbol{\gamma}_{\boldsymbol{k}}^{m}$ as defined in Eq. (60) is an interband coherence effect mediated by an effective offdiagonal spin operator defined in Eq. (56).

The side-jump contribution is given by

$$
\left\langle s_{x}\right\rangle^{\mathrm{sj}}=\sum_{\boldsymbol{k}} s_{\boldsymbol{k}, x}^{++} n_{E \boldsymbol{k}}^{(\mathrm{sj})++} .
$$

Using the spin expectation values above and the density function given by Eq. (81), we find that the side-jump contribution to the spin density reads

$$
\langle\boldsymbol{s}\rangle^{\mathrm{sj}}=\frac{e \hbar v_{F} \rho\left(\epsilon_{F}\right) M}{\epsilon_{F}^{2}} \frac{\left(1-\xi_{F}^{2}\right)}{\left(1+3 \xi_{F}^{2}\right)} \boldsymbol{E} .
$$

This term doubles the contribution of Eq. (99). This is the counterpart of the anomalous distribution function introduced in the semiclassical theory [92] and also calculated in Eq. (82) for the anomalous Hall conductivity.

The skew scattering contribution takes the form

$$
\langle\boldsymbol{s}\rangle^{\text {skew }}=\sum_{\boldsymbol{k}}\left[\boldsymbol{s}_{\boldsymbol{k}, x}^{++} n_{E \boldsymbol{k}}^{(\mathrm{sk})++}+\boldsymbol{s}_{\boldsymbol{k}}^{--} n_{E \boldsymbol{k}}^{(\mathrm{sk})--}\right] .
$$

Using the diagonal spin expectation values and the distribution function found in Eq. (84), we find for the skew scattering contribution to the spin density

$$
\langle\boldsymbol{s}\rangle^{\mathrm{sk}}=\frac{e \hbar \rho\left(\epsilon_{F}\right) v_{F} \xi_{F}}{\lambda_{F}} \frac{3\left(1-\xi_{F}^{2}\right)^{2}}{2\left(1+3 \xi_{F}^{2}\right)^{2}} \boldsymbol{E} .
$$

This is the counterpart of the skew scattering term in the anomalous Hall conductivity calculated in Eq. (85). 
Finally, the total spin density of the system then reads

$$
\begin{aligned}
\langle\boldsymbol{s}\rangle= & -e \tau v_{F} \rho\left(\epsilon_{F}\right) \frac{\left(1-\xi_{F}^{2}\right)}{\left(1+3 \xi_{F}^{2}\right)} \hat{z} \times \boldsymbol{E} \\
& +4 e \hbar v_{F} \rho\left(\epsilon_{F}\right) \frac{M}{\epsilon_{F}^{2}} \frac{\left(1+\xi_{F}^{2}\right)}{\left(1+3 \xi_{F}^{2}\right)^{2}} \boldsymbol{E} .
\end{aligned}
$$

The spin-orbit torque is defined as $\boldsymbol{\tau}=(2 M / \hbar) \boldsymbol{m} \times\langle\boldsymbol{s}\rangle$, where $\boldsymbol{m}$ is a unit vector in the direction of magnetization that we took here as $\boldsymbol{m}=\hat{\boldsymbol{z}}$. Restoring the factor of $\hbar / 2$ in the spin matrix elements and substituting the density of states $\rho\left(\epsilon_{F}\right)=$ $\epsilon_{F} / 2 \pi \hbar^{2} v_{F}^{2}$, the spin-orbit torque is finally given by

$$
\begin{aligned}
\boldsymbol{\tau}= & -\frac{e \tau \epsilon_{F} M \rho\left(\epsilon_{F}\right)}{2 \pi \hbar^{2} v_{F}} \frac{\left(1-\xi_{F}^{2}\right)}{\left(1+3 \xi_{F}^{2}\right)} \boldsymbol{m} \times(\hat{z} \times \boldsymbol{E}) \\
& +\frac{2 e}{\pi \hbar v_{F}} \frac{M^{2}}{\epsilon_{F}} \frac{\left(1+\xi_{F}^{2}\right)}{\left(1+3 \xi_{F}^{2}\right)^{2}} \boldsymbol{m} \times \boldsymbol{E},
\end{aligned}
$$

in agreement with previous results [90,98]. A peculiarity of topological insulators is that the velocity operator is directly related to the spin operator as $\hat{\boldsymbol{v}}=-v_{F} \hat{\boldsymbol{z}} \times \sigma$. Then the current density is $\langle\boldsymbol{j}\rangle=-e\langle\hat{\boldsymbol{v}}\rangle$, which implies that the anomalous Hall conductivity and the spin density are related by $\sigma_{y x}=J_{y} / E_{x}=$ $e v_{F}\left\langle s_{x}\right\rangle$. This fact can indeed be verified from Eqs. (86) and (104).

\section{CONCLUSIONS AND OUTLOOK}

We have demonstrated that the semiclassical dynamics of electrons in disordered solids can be determined using linear response theory by taking the density matrix and quantum Liouville equation as the starting point. This results in a disorder-dependent correction to the semiclassical equation of motion for the carrier position, which we have termed the extrinsic velocity $\boldsymbol{\beta}_{\boldsymbol{k}}^{m}$ and which accounts for the effect of disorder on the carrier velocity after many collisions. In analogy to the extrinsic velocity, an extrinsic correction to the spin expectation value $\boldsymbol{\gamma}_{k}^{m}$ is also present, which accounts for spin rotations during scattering events. This is accompanied by an intrinsic, electric-field-dependent nonequilibrium correction to the spin expectation value, which is analogous to the anomalous velocity present in the semiclassical equations of motion. At the same time, the Boltzmann equation must be solved up to subleading order in the disorder strength. The collision integral in the Boltzmann equation includes an electric-field-dependent correction which is analogous to the side-jump scattering term, as well as an additional correction analogous to the skew scattering term in systems with intrinsic spin-orbit interactions. We have applied this theory to describe the anomalous Hall effect and spin-orbit torques in topological insulators, obtaining exact agreement with quantum mechanical results using the Kubo formula.

The general prescription we have formulated in this paper can be straightforwardly generalized to include extrinsic spinorbit and magnetic impurity scattering, as well as magnetic fields, which, however, require more effort since the description relies on the Wigner function. Our prescription paves the way towards a systematic semiclassical picture encompassing a host of effects that conventionally lie beyond the purview of semiclassical theory: spin relaxation, disorder effects beyond the Born approximation, such as weak localization, electronelectron interactions in the mean-field approximation, Kondo physics in magnetic systems, and the nonlinear electromagnetic response.

\section{ACKNOWLEDGMENTS}

D.C. is supported by the Australian Research Council Future Fellowship FT190100062. We would like to thank Leonid Glazman, Joel Moore, Aydin Keser, Cong Xiao, and Dmitry Efimkin for a series of educational discussions.
[1] G. Sundaram and Q. Niu, Wave-packet dynamics in slowly perturbed crystals: Gradient corrections and Berry-phase effects, Phys. Rev. B 59, 14915 (1999).

[2] N. W. Ashcroft and N. D. Mermin, Solid State Physics (HoltSaunders, New York, 1976).

[3] T. Jungwirth, Q. Niu, and A. H. MacDonald, Anomalous Hall Effect in Ferromagnetic Semiconductors, Phys. Rev. Lett. 88, 207208 (2002).

[4] R. Matsumoto and S. Murakami, Theoretical Prediction of a Rotating Magnon Wave Packet in Ferromagnets, Phys. Rev. Lett. 106, 197202 (2011).

[5] R. Cheng, S. Okamoto, and D. Xiao, Spin Nernst Effect of Magnons in Collinear Antiferromagnets, Phys. Rev. Lett. 117, 217202 (2016).

[6] Y. Gao and D. Xiao, Nonreciprocal Directional Dichroism Induced by the Quantum Metric Dipole, Phys. Rev. Lett. 122, 227402 (2019).

[7] X. Zhang, Y. Zhang, S. Okamoto, and D. Xiao, Thermal Hall Effect Induced by Magnon-Phonon Interactions, Phys. Rev. Lett. 123, 167202 (2019).
[8] R. Shindou and K.-I. Imura, Noncommutative geometry and non-Abelian Berry phase in the wave-packet dynamics of Bloch electrons, Nucl. Phys. B 720, 399 (2005).

[9] E. Dumitrescu, C. Zhang, D. C. Marinescu, and S. Tewari, Topological thermoelectric effects in spin-orbit coupled electron- and hole-doped semiconductors, Phys. Rev. B 85, 245301 (2012).

[10] D. T. Son and B. Z. Spivak, Chiral anomaly and classical negative magnetoresistance of Weyl metals, Phys. Rev. B 88, 104412 (2013).

[11] J. C. W. Song, G. Refael, and P. A. Lee, Linear magnetoresistance in metals: Guiding center diffusion in a smooth random potential, Phys. Rev. B 92, 180204(R) (2015).

[12] S. A. Yang, H. Pan, and F. Zhang, Chirality-Dependent Hall Effect in Weyl Semimetals, Phys. Rev. Lett. 115, 156603 (2015).

[13] Y. D. Lensky, J. C. W. Song, P. Samutpraphoot, and L. S. Levitov, Topological Valley Currents in Gapped Dirac Materials, Phys. Rev. Lett. 114, 256601 (2015). 
[14] M.-C. Chang and M.-F. Yang, Chiral magnetic effect in a twoband lattice model of Weyl semimetal, Phys. Rev. B 91, 115203 (2015).

[15] E. V. Gorbar, V. A. Miransky, I. A. Shovkovy, and P. O. Sukhachov, Non-Abelian properties of electron wave packets in the Dirac semimetals $A_{3} \mathrm{Bi}(A=\mathrm{Na}, \mathrm{K}, \mathrm{Rb})$, Phys. Rev. B 98, 045203 (2018).

[16] K. Misaki, S. Miyashita, and N. Nagaosa, Diffusive real-time dynamics of a particle with Berry curvature, Phys. Rev. B 97, 075122 (2018).

[17] Y. Araki, Strain-induced nonlinear spin Hall effect in topological Dirac semimetal, Sci. Rep. 8, 15236 (2018).

[18] A. Alexandradinata and L. Glazman, Semiclassical theory of Landau levels and magnetic breakdown in topological metals, Phys. Rev. B 97, 144422 (2018).

[19] K. Das and A. Agarwal, Linear magnetochiral transport in tilted type-I and type-II Weyl semimetals, Phys. Rev. B 99, 085405 (2019).

[20] S. Nandy, A. Taraphder, and S. Tewari, Planar thermal Hall effect in Weyl semimetals, Phys. Rev. B 100, 115139 (2019).

[21] Z. Hou, Y.-F. Zhou, N.-X. Yang, and Q.-F. Sun, Chiralitydependent electron transport in Weyl semimetal $\mathrm{p}-\mathrm{n}-\mathrm{p}$ junctions, Commun. Phys. 2, 86 (2019).

[22] P. Zhu, T. L. Hughes, and A. Alexandradinata, Quantized surface magnetism and higher-order topology: Application to the Hopf insulator, Phys. Rev. B 103, 014417 (2021).

[23] T. Yokoyama, Absence of Hall effect due to Berry curvature in phase space, Sci. Rep. 11, 12065 (2021).

[24] Z. Wang, L. Dong, C. Xiao, and Q. Niu, Berry Curvature Effects on Quasiparticle Dynamics in Superconductors, Phys. Rev. Lett. 126, 187001 (2021).

[25] K. Das and A. Agarwal, Intrinsic Hall conductivities induced by the orbital magnetic moment, Phys. Rev. B 103, 125432 (2021).

[26] S. Chaudhary, C. Knapp, and G. Refael, Anomalous exciton transport in response to a uniform in-plane electric field, Phys. Rev. B 103, 165119 (2021).

[27] S. Bhowal and G. Vignale, Orbital Hall effect as an alternative to valley Hall effect in gapped graphene, Phys. Rev. B 103, 195309 (2021).

[28] J. E. Moore and J. Orenstein, Confinement-Induced Berry Phase and Helicity-Dependent Photocurrents, Phys. Rev. Lett. 105, 026805 (2010).

[29] I. Sodemann and L. Fu, Quantum Nonlinear Hall Effect Induced by Berry Curvature Dipole in Time-Reversal Invariant Materials, Phys. Rev. Lett. 115, 216806 (2015).

[30] T. Morimoto, S. Zhong, J. Orenstein, and J. E. Moore, Semiclassical theory of nonlinear magneto-optical responses with applications to topological Dirac/Weyl semimetals, Phys. Rev. B 94, 245121 (2016).

[31] C. Zeng, S. Nandy, A. Taraphder, and S. Tewari, Nonlinear Nernst effect in bilayer $\mathrm{WTe}_{2}$, Phys. Rev. B 100, 245102 (2019).

[32] L. E. Golub, E. L. Ivchenko, and B. Spivak, Semiclassical theory of the circular photogalvanic effect in gyrotropic systems, Phys. Rev. B 102, 085202 (2020).

[33] E. J. König and A. Levchenko, Quantum kinetics of anomalous and nonlinear Hall effects in topological semimetals, Ann. Phys. (Amsterdam) 435, 168492 (2021).

[34] X. Wang, J. R. Yates, I. Souza, and D. Vanderbilt, Ab initio calculation of the anomalous Hall conductivity by Wannier interpolation, Phys. Rev. B 74, 195118 (2006).
[35] X. Wang, D. Vanderbilt, J. R. Yates, and I. Souza, Fermi-surface calculation of the anomalous Hall conductivity, Phys. Rev. B 76, 195109 (2007)

[36] M. Gradhand, D. V. Fedorov, F. Pientka, P. Zahn, I. Mertig, and B. L. Györffy, First-principle calculations of the Berry curvature of Bloch states for charge and spin transport of electrons, J. Phys.: Condens. Matter 24, 213202 (2012).

[37] Y. He, J. Moore, and C. M. Varma, Berry phase and anomalous Hall effect in a three-orbital tight-binding Hamiltonian, Phys. Rev. B 85, 155106 (2012).

[38] Y. Chen, D. L. Bergman, and A. A. Burkov, Weyl fermions and the anomalous Hall effect in metallic ferromagnets, Phys. Rev. B 88, 125110 (2013).

[39] R. Bianco, R. Resta, and I. Souza, How disorder affects the Berry-phase anomalous Hall conductivity: A reciprocal-space analysis, Phys. Rev. B 90, 125153 (2014).

[40] H. Chen, Q. Niu, and A. H. MacDonald, Anomalous Hall Effect Arising from Noncollinear Antiferromagnetism, Phys. Rev. Lett. 112, 017205 (2014).

[41] T. Olsen and I. Souza, Valley Hall effect in disordered monolayer $\mathrm{MoS}_{2}$ from first principles, Phys. Rev. B 92, 125146 (2015).

[42] W. Feng, C.-C. Liu, G.-B. Liu, J.-J. Zhou, and Y. Yao, Firstprinciples investigations on the Berry phase effect in spin-orbit coupling materials, Comput. Mater. Sci 112, 428 (2016).

[43] X. Dai, Z. Z. Du, and H.-Z. Lu, Negative Magnetoresistance without Chiral Anomaly in Topological Insulators, Phys. Rev. Lett. 119, 166601 (2017).

[44] J. H. J. Martiny, K. Kaasbjerg, and A.-P. Jauho, Tunable valley Hall effect in gate-defined graphene superlattices, Phys. Rev. B 100, 155414 (2019).

[45] C. Wuttke, F. Caglieris, S. Sykora, F. Scaravaggi, A. U. B. Wolter, K. Manna, V. Süss, C. Shekhar, C. Felser, B. Büchner, and C. Hess, Berry curvature unravelled by the anomalous Nernst effect in $\mathrm{Mn}_{3} \mathrm{Ge}$, Phys. Rev. B 100, 085111 (2019).

[46] S. Du, P. Tang, J. Li, Z. Lin, Y. Xu, W. Duan, and A. Rubio, Berry curvature engineering by gating two-dimensional antiferromagnets, Phys. Rev. Res. 2, 022025(R) (2020).

[47] W.-Y. He, D. Goldhaber-Gordon, and K. T. Law, Giant orbital magnetoelectric effect and current-induced magnetization switching in twisted bilayer graphene, Nat. Commun. 11, 1650 (2020).

[48] W.-Y. He and K. T. Law, Superconducting orbital magnetoelectric effect and its evolution across the superconductor-normal metal phase transition, Phys. Rev. Res. 3, L032012 (2021).

[49] M.-C. Chang and Q. Niu, Berry curvature, orbital moment, and effective quantum theory of electrons in electromagnetic fields, J. Phys.: Condens. Matter 20, 193202 (2008).

[50] D. Culcer, A. Sekine, and A. H. MacDonald, Interband coherence response to electric fields in crystals: Berry-phase contributions and disorder effects, Phys. Rev. B 96, 035106 (2017).

[51] T. Stedman, C. Timm, and L. M. Woods, Multiband effects in equations of motion of observables beyond the semiclassical approach, New J. Phys. 21, 103007 (2019).

[52] T. Stedman and L. M. Woods, Transport theory within a generalized Boltzmann equation for multiband wave packets, Phys. Rev. Res. 2, 033086 (2020).

[53] D. Xiao, M.-C. Chang, and Q. Niu, Berry phase effects on electronic properties, Rev. Mod. Phys. 82, 1959 (2010). 
[54] E. G. Mishchenko, A. V. Shytov, and B. I. Halperin, Spin Current and Polarization in Impure Two-Dimensional Electron Systems with Spin-Orbit Coupling, Phys. Rev. Lett. 93, 226602 (2004).

[55] J.-i. Inoue, G. E. W. Bauer, and L. W. Molenkamp, Suppression of the persistent spin Hall current by defect scattering, Phys. Rev. B 70, 041303(R) (2004).

[56] R. Raimondi and P. Schwab, Spin-Hall effect in a disordered 2D electron-system, Phys. Rev. B 71, 033311 (2005).

[57] O. V. Dimitrova, Spin-Hall conductivity in a two-dimensional Rashba electron gas, Phys. Rev. B 71, 245327 (2005).

[58] A. Khaetskii, Nonexistence of Intrinsic Spin Currents, Phys. Rev. Lett. 96, 056602 (2006).

[59] J.-i. Inoue, T. Kato, Y. Ishikawa, H. Itoh, G. E. W. Bauer, and L. W. Molenkamp, Vertex Corrections to the Anomalous Hall Effect in Spin-Polarized Two-Dimensional Electron Gases with a Rashba Spin-Orbit Interaction, Phys. Rev. Lett. 97, 046604 (2006).

[60] D. Culcer and R. Winkler, Generation of Spin Currents and Spin Densities in Systems with Reduced Symmetry, Phys. Rev. Lett. 99, 226601 (2007).

[61] R. Karplus and J. Luttinger, Hall Effect in Ferromagnetics, Phys. Rev. 95, 1154 (1954).

[62] J. M. Luttinger, Theory of the Hall Effect in Ferromagnetic Substances, Phys. Rev. 112, 739 (1958).

[63] J. Smit, The spontaneous hall effect in ferromagnetics II, Physica 24, 39 (1958).

[64] L. Berger, Side-Jump Mechanism for the Hall Effect of Ferromagnets, Phys. Rev. B 2, 4559 (1970).

[65] P. Nozières and C. Lewiner, A simple theory of the anomalous hall effect in semiconductors, J. Phys. (Paris) 34, 901 (1973).

[66] A. A. Kovalev, J. Sinova, and Y. Tserkovnyak, Anomalous Hall Effect in Disordered Multiband Metals, Phys. Rev. Lett. 105, 036601 (2010).

[67] S. A. Yang, H. Pan, Y. Yao, and Q. Niu, Scattering universality classes of side jump in the anomalous Hall effect, Phys. Rev. B 83, 125122 (2011).

[68] I. A. Ado, I. A. Dmitriev, P. M. Ostrovsky, and M. Titov, Anomalous Hall effect with massive Dirac fermions, Europhys. Lett. 111, 37004 (2015).

[69] I. A. Ado, I. A. Dmitriev, P. M. Ostrovsky, and M. Titov, Anomalous Hall Effect in a 2D Rashba Ferromagnet, Phys. Rev. Lett. 117, 046601 (2016).

[70] I. A. Ado, I. A. Dmitriev, P. M. Ostrovsky, and M. Titov, Sensitivity of the anomalous Hall effect to disorder correlations, Phys. Rev. B 96, 235148 (2017).

[71] T. Rauch, T. Olsen, D. Vanderbilt, and I. Souza, Geometric and nongeometric contributions to the surface anomalous Hall conductivity, Phys. Rev. B 98, 115108 (2018).

[72] A. C. Keser, R. Raimondi, and D. Culcer, Sign Change in the Anomalous Hall Effect and Strong Transport Effects in a 2D Massive Dirac Metal Due to Spin-Charge Correlated Disorder, Phys. Rev. Lett. 123, 126603 (2019).

[73] J. Shen, Q. Yao, Q. Zeng, H. Sun, X. Xi, G. Wu, W. Wang, B. Shen, Q. Liu, and E. Liu, Local Disorder-Induced Elevation of Intrinsic Anomalous Hall Conductance in an ElectronDoped Magnetic Weyl Semimetal, Phys. Rev. Lett. 125, 086602 (2020).
[74] N. A. Sinitsyn, Q. Niu, J. Sinova, and K. Nomura, Disorder effects in the anomalous Hall effect induced by Berry curvature, Phys. Rev. B 72, 045346 (2005).

[75] N. A. Sinitsyn, A. H. MacDonald, T. Jungwirth, V. K. Dugaev, and J. Sinova, Anomalous Hall effect in a two-dimensional Dirac band: The link between the Kubo-Streda formula and the semiclassical Boltzmann equation approach, Phys. Rev. B 75, 045315 (2007).

[76] N. A. Sinitsyn, Semiclassical theories of the anomalous Hall effect, J. Phys.: Condens. Matter 20, 023201 (2007).

[77] C. Xiao and Q. Niu, Semiclassical theory of spin-orbit torques in disordered multiband electron systems, Phys. Rev. B 96, 045428 (2017).

[78] C. Xiao, Y. Liu, Z. Yuan, S. A. Yang, and Q. Niu, Temperature dependence of the side-jump spin Hall conductivity, Phys. Rev. B 100, 085425 (2019).

[79] F. T. Vasko and O. E. Raichev, Quantum Kinetic Theory and Applications: Electrons, Photons, Phonons, 1st ed. (Springer, New York, 2005).

[80] A. A. Burkov, A. S. Nunez, and A. H. MacDonald, Theory of spin-charge-coupled transport in a two-dimensional electron gas with Rashba spin-orbit interactions, Phys. Rev. B 70, 155308 (2004).

[81] N. A. Sinitsyn, Q. Niu, and A. H. MacDonald, Coordinate shift in the semiclassical Boltzmann equation and the anomalous Hall effect, Phys. Rev. B 73, 075318 (2006).

[82] N. Nagaosa, J. Sinova, S. Onoda, A. H. MacDonald, and N. P. Ong, Anomalous Hall effect, Rev. Mod. Phys. 82, 1539 (2010).

[83] A. Sekine, D. Culcer, and A. H. MacDonald, Quantum kinetic theory of the chiral anomaly, Phys. Rev. B 96, 235134 (2017).

[84] N. Silberstein, J. Behrends, M. Goldstein, and R. Ilan, Berry connection induced anomalous wave-packet dynamics in nonHermitian systems, Phys. Rev. B 102, 245147 (2020).

[85] A. Marrazzo and R. Resta, Locality of the anomalous Hall conductivity, Phys. Rev. B 95, 121114(R) (2017).

[86] M. F. Lapa and T. L. Hughes, Semiclassical wave packet dynamics in nonuniform electric fields, Phys. Rev. B 99, 121111(R) (2019).

[87] M. W.-Y. Tu, C. Li, and W. Yao, Theory of wave-packet transport under narrow gaps and spatial textures: Nonadiabaticity and semiclassicality, Phys. Rev. B 102, 045423 (2020).

[88] V. Kozii, A. Avdoshkin, S. Zhong, and J. E. Moore, Intrinsic Anomalous Hall Conductivity in a Nonuniform Electric Field, Phys. Rev. Lett. 126, 156602 (2021).

[89] D. Culcer, E. M. Hankiewicz, G. Vignale, and R. Winkler, Side jumps in the spin Hall effect: Construction of the Boltzmann collision integral, Phys. Rev. B 81, 125332 (2010).

[90] P. B. Ndiaye, C. A. Akosa, M. H. Fischer, A. Vaezi, E.-A. Kim, and A. Manchon, Dirac spin-orbit torques and charge pumping at the surface of topological insulators, Phys. Rev. B 96, 014408 (2017).

[91] P. B. Allen, New method for solving Boltzmann's equation for electrons in metals, Phys. Rev. B 17, 3725 (1978).

[92] N. A. Sinitsyn, J. E. Hill, H. Min, J. Sinova, and A. H. MacDonald, Charge and Spin Hall Conductivity in Metallic Graphene, Phys. Rev. Lett. 97, 106804 (2006).

[93] H. Bruus and K. Flensberg, Many-Body Quantum Theory in Condensed Matter Physics: An Introduction (Oxford University Press, New York, 2004). 
[94] D. Culcer, Transport in three-dimensional topological insulators: Theory and experiment, Phys. E (Amsterdam) 44, 860 (2012).

[95] E. J. König, P. M. Ostrovsky, M. Dzero, and A. Levchenko, Anomalous Hall effect on the surface of topological Kondo insulators, Phys. Rev. B 94, 041403(R) (2016).

[96] D. Culcer and S. Das Sarma, Anomalous Hall response of topological insulators, Phys. Rev. B 83, 245441 (2011).
[97] V. Edelstein, Spin polarization of conduction electrons induced by electric current in two-dimensional asymmetric electron systems, Solid State Commun. 73, 233 (1990).

[98] A. Sakai and H. Kohno, Spin torques and charge transport on the surface of topological insulator, Phys. Rev. B 89, 165307 (2014). 